\title{
A PRIORI ERROR ESTIMATES FOR FINITE ELEMENT DISCRETIZATIONS OF A SHAPE OPTIMIZATION PROBLEM
}

\author{
BERNHARD KINIGER ${ }^{1}$ AND BORIS VEXLER ${ }^{1}$
}

\begin{abstract}
In this paper we consider a model shape optimization problem. The state variable solves an elliptic equation on a domain with one part of the boundary described as the graph of a control function. We prove higher regularity of the control and develop a priori error analysis for the finite element discretization of the shape optimization problem under consideration. The derived a priori error estimates are illustrated on two numerical examples.
\end{abstract}

Mathematics Subject Classification. 49Q10, 49M25, 65M15, 65M60.

Received November 14, 2011. Revised March 5, 2013.

Published online October 7, 2013.

\section{INTRODUCTION}

In this paper we consider the following shape optimization problem governed by a linear elliptic equation:

$$
\min J(q, u)=\frac{1}{2}\left\|u-u_{d}^{q}\right\|_{\mathrm{L}^{2}\left(\Omega_{q}\right)}^{2}+\frac{\alpha}{2}\left\|q^{\prime \prime}\right\|_{\mathrm{L}^{2}((0,1))}^{2},
$$

subject to

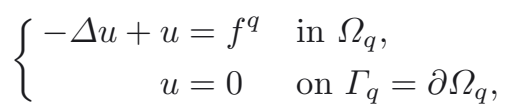

where the domain $\Omega_{q}$ is basically the unit square with one "moving" side given as the graph of the control function $q$, see Figure 1. The data functions $u_{d}^{q}$ and $f^{q}$ are restrictions of functions $u_{d}$ and $f$ defined on a sufficiently large (holding-all) domain $\hat{\Omega}$. The precise formulation of the problem including a functional analytic setting is presented in Section 1.

Similar shape optimization problems, where the unknown part of the boundary is parameterized as a graph of a function, are considered in various publications, see, e.g., $[13,14,19,25]$. The problem formulation in these publications involves a bound on an appropriate norm of $q$. Our formulation utilizes a Tikhonov-type term $\left\|q^{\prime \prime}\right\|_{L^{2}((0,1))}^{2}$ instead.

The main contribution of this paper is an a priori error analysis for a finite element discretization of the shape optimization problem under consideration. The control variable is discretized by Hermite finite elements allowing

Keywords and phrases. Shape optimization, existence and convergence of approximate solutions, error estimates, finite elements.

1 Lehrstuhl für Optimale Steuerung, Technische Universität München, Fakultät für Mathematik, Boltzmannstraße 3, 85748

Garching b. München, Germany. kiniger@ma.tum.de; vexler@ma.tum.de 


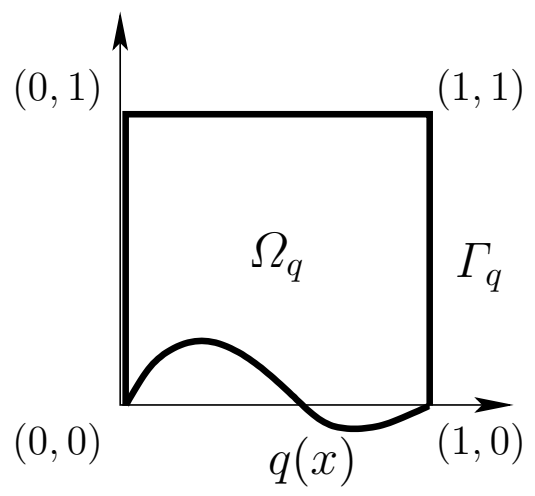

FiguRE 1. Domain $\Omega_{q}$.

for conforming discretization of the control space $\mathrm{H}^{2}((0,1)) \cap \mathrm{H}_{0}^{1}((0,1))$. The corresponding discretization parameter is called $\sigma$. The state variable is discretized using usual (bi)linear finite elements with the discretization parameter $h$. The main result (see Thm. 3.2) is the estimate

$$
\left\|\bar{q}-\bar{q}_{\sigma, h}\right\|_{\mathrm{H}^{2}((0,1))} \leq c\left(\sigma^{2}+h^{2}\right),
$$

where $\bar{q}$ is a local solution of the optimization problem fulfilling a second order sufficient optimality condition and $\left(\bar{q}_{\sigma, h}\right)((\sigma, h) \rightarrow 0)$ is a sequence of local solutions of the discretized problem converging to $\bar{q}$. The existence of such a sequence is also shown.

There are some published results on discretization of shape optimizations problems. In $[13,14]$ and in $[8,9]$ convergence of discrete solutions to a continuous one is investigated. To our best knowledge only the paper [11] provides convergence results including the rate of convergence for a discretized shape optimization problem using a fast wavelet boundary element method for the state equation, where the discretization of the state is treated as a consistency error and is not restricted to boundary integral equation methods.

The paper is organized as follows: In the next section we discuss a precise formulation of the shape optimization problem under consideration, show the existence of at least one globally optimal solution applying the techniques similar to [13], reformulate the problem using a transformation to a reference domain $\Omega_{0}$ and present optimality conditions. Section 2 is devoted to the discretization. The control variable is discretized by Hermite finite elements of third order on $(0,1)$, leading to the semidiscrete problem. Then the state variable is discretized by (bi)linear finite elements on the reference domain $\Omega_{0}$ resulting in a full discretization of the problem. We show that both the semidiscrete and the discrete problems possess solutions and discuss their convergence for $(\sigma, h) \rightarrow 0$. In Section 3 we prove that any optimal solution $\bar{q}$ possesses higher regularity, i.e. $\bar{q} \in \mathrm{H}^{4}((0,1))$. This result is essential for deriving a priori error estimates. Due to the fact that the considered optimization problem is not convex in general, we have to deal with second order sufficient optimality conditions and adapt the technique from [6]. We first provide error estimates for the discretization error between a solution of the continuous problem and the corresponding solution of the semidiscrete one and then for the error between a semidiscrete and a fully discrete solution. In Section 4 we present numerical examples illustrating our results.

Throughout the paper, $c$ and $c_{i}$ shall always denote generic constants which are - if not stated otherwise independent of $\sigma$ and $h$, but may depend on $\alpha$, and have different values on different appearances. With $\mathrm{L}^{p}(\Omega)$ and $\mathrm{W}^{k, p}(\Omega)$ we will denote the Lebesgue and the Sobolev spaces on the domain $\Omega$, respectively. For $p=2$ we use the standard notation $\mathrm{H}^{k}(\Omega)=\mathrm{W}^{k, 2}(\Omega)$. For an arbitrary Hilbert space $X$ we will denote its scalar product with $(\cdot, \cdot)_{X}$ and the corresponding norm with $\|\cdot\|_{X}$, whereas for $X=\mathrm{L}^{2}\left(\Omega_{0}\right)$ we omit the subindex. The set of all $m$-times continuously differentiable functions whose $m$ th derivative is Hölder-continuous with index $\alpha$ will be denoted by $\mathrm{C}^{m, \alpha}(\bar{\Omega})$. For functions $q$ defined on an interval $I \subset \mathbb{R}, q^{\prime}$ and $q^{\prime \prime}$ shall denote the first and second order weak derivative, respectively. 


\section{Optimization problem}

\subsection{Problem formulation and existence result}

In this section we first describe the shape optimization problem under consideration. The control variable $q$ from the control space $Q=\mathrm{H}^{2}(I) \cap \mathrm{H}_{0}^{1}(I)$ with $I=(0,1)$ characterizes the domain $\Omega_{q}$ through:

$$
\Omega_{q}=\left\{(x, y) \in \mathbb{R}^{2} \mid x \in I, y \in(q(x), 1)\right\} .
$$

To exclude a possible degeneracy of the domain $\Omega_{q}$ we fix $0<\varepsilon<1$ and define the set

$$
\bar{Q}^{\mathrm{ad}}=\{q \in Q \mid q(x) \leq 1-\varepsilon \text { for all } x \in I\} .
$$

For each $q \in \bar{Q}^{\text {ad }}$ the domain $\Omega_{q}$ is a Lipschitz domain, which allows for the definition of the state variable $u \in \mathrm{H}_{0}^{1}\left(\Omega_{q}\right)$ being the weak solution of the state equation

$$
\left\{\begin{aligned}
-\Delta u+u=f^{q} & \text { in } \Omega_{q} \\
u=0 & \text { on } \Gamma_{q}=\partial \Omega_{q} .
\end{aligned}\right.
$$

The shape optimization problem is then given as:

$$
\text { Minimize } J(q, u)=\frac{1}{2}\left\|u-u_{d}^{q}\right\|_{\mathrm{L}^{2}\left(\Omega_{q}\right)}^{2}+\frac{\alpha}{2}\left\|q^{\prime \prime}\right\|_{\mathrm{L}^{2}(I)}^{2}, \quad q \in \bar{Q}^{\mathrm{ad}}, u \in \mathrm{H}_{0}^{1}\left(\Omega_{q}\right),
$$

subject to (1.2).

We define the solution operator $\tilde{S}$, which assigns to each $q \in \bar{Q}^{\text {ad }}$ the unique solution $\tilde{u}(q)=\tilde{S}(q)$ of $(1.2)$. This allows to introduce the reduced cost functional $j: \bar{Q}^{\text {ad }} \mapsto \mathbb{R}$ by

$$
j(q)=J(q, \tilde{S}(q)) .
$$

In order to prove the existence of an optimal solution to (1.3), we need bounds in the full $\mathrm{H}^{2}(I)$-norm.

Lemma 1.1. On $Q$, the $\mathrm{H}^{2}(I)$-seminorm is equivalent to the full $\mathrm{H}^{2}(I)$-norm.

Proof. Let $q \in Q$ be arbitrary. As $Q \subset \mathrm{H}_{0}^{1}(I)$ we know that there exists $c_{p}>0$ such that $\|q\|_{\mathrm{L}^{2}(I)} \leq c_{p} \cdot\left\|q^{\prime}\right\|_{\mathrm{L}^{2}(I)}$. Furthermore,

$$
\left\|q^{\prime}\right\|_{\mathrm{L}^{2}(I)}^{2}=\int_{0}^{1} q^{\prime}(x)^{2} \mathrm{~d} x=-\int_{0}^{1} q(x) q^{\prime \prime}(x) \mathrm{d} x \leq\|q\|_{\mathrm{L}^{2}(I)} \cdot\left\|q^{\prime \prime}\right\|_{\mathrm{L}^{2}(I)} \leq c_{p} \cdot\left\|q^{\prime}\right\|_{\mathrm{L}^{2}(I)} \cdot\left\|q^{\prime \prime}\right\|_{\mathrm{L}^{2}(I)},
$$

which leads to

$$
\left\|q^{\prime}\right\|_{\mathrm{L}^{2}(I)} \leq c_{p} \cdot\left\|q^{\prime \prime}\right\|_{\mathrm{L}^{2}(I)}
$$

As a result,

$$
\|q\|_{\mathrm{H}^{2}(I)}^{2}=\|q\|_{\mathrm{L}^{2}(I)}^{2}+\left\|q^{\prime}\right\|_{\mathrm{L}^{2}(I)}^{2}+\left\|q^{\prime \prime}\right\|_{\mathrm{L}^{2}(I)}^{2} \leq\left(c_{p}^{4}+c_{p}^{2}+1\right) \cdot\left\|q^{\prime \prime}\right\|_{\mathrm{L}^{2}(I)}^{2} .
$$

In order to bound $\bar{Q}^{\text {ad }}$ in $\mathrm{H}^{2}(I)$, we set $q_{0}=0 \in \bar{Q}^{\text {ad }}$. A necessary condition for $q \in \bar{Q}^{\text {ad }}$ to be a solution to $(1.3)$ is

$$
j(q) \leq j\left(q_{0}\right)
$$


which is

$$
\frac{1}{2}\left\|S(q)-u_{d}^{q}\right\|_{\mathrm{L}^{2}\left(\Omega_{q}\right)}^{2}+\frac{\alpha}{2}\left\|q^{\prime \prime}\right\|_{\mathrm{L}^{2}(I)}^{2} \leq j\left(q_{0}\right)
$$

This implies

$$
\left\|q^{\prime \prime}\right\|_{\mathrm{L}^{2}(I)}^{2} \leq \frac{2}{\alpha}\left(j\left(q_{0}\right)-\frac{1}{2}\left\|S(q)-u_{d}^{q}\right\|_{\mathrm{L}^{2}\left(\Omega_{q}\right)}^{2}\right) \leq \frac{2}{\alpha} j\left(q_{0}\right) .
$$

Together with Lemma 1.1 this shows that there exists a constant $\tilde{C}=\tilde{C}(\alpha)>0$, such that the search for a minima can be restricted to the set

$$
Q^{\mathrm{ad}}=\left\{q \in \bar{Q}^{\mathrm{ad}} \mid\|q\|_{\mathrm{H}^{2}(I)} \leq \tilde{C}\right\},
$$

which is also bounded in $\mathrm{C}^{1}(\bar{I})$.

Throughout we assume for the data

$$
u_{d}^{q}=\left.u_{d}\right|_{\Omega_{q}}, f^{q}=\left.f\right|_{\Omega_{q}}, \quad \text { with } u_{d}, f \in \mathrm{C}^{2,1}(\hat{\Omega}),
$$

where $\hat{\Omega}$ is the all-holding domain with $\Omega_{q} \subset \hat{\Omega}$ for all $q \in Q^{\text {ad }}$. We will therefore just write $f$ and $u_{d}$ instead of $f^{q}$ and $u_{d}^{q}$.

For $q \in Q^{\text {ad }}$ and $v \in \mathrm{H}_{0}^{1}\left(\Omega_{q}\right)$ we define an extension $\tilde{v} \in \mathrm{H}_{0}^{1}(\hat{\Omega})$ by

$$
\tilde{v}(x)= \begin{cases}v(x) & \text { if } x \in \Omega_{q}, \\ 0 & \text { else. }\end{cases}
$$

If $v_{n} \in \mathrm{H}_{0}^{1}\left(\Omega_{q_{n}}\right)$ for $q_{n} \in Q^{\text {ad }}$, then the sequence $\left(v_{n}\right)_{n \in \mathbb{N}}$ is said to (weakly) converge to an element $v \in \mathrm{H}_{0}^{1}\left(\Omega_{q}\right)$ if the extended functions $\tilde{v}_{n}$ (weakly) converge to $\tilde{v}$ in $\mathrm{H}_{0}^{1}(\hat{\Omega})$.

The following proposition states the continuity of the solution operator $\tilde{S}$, see [13] for the proof.

Proposition 1.2. Let $q_{n}, q \in Q^{a d}, q_{n} \rightarrow q$ in $\mathrm{L}^{\infty}(I)$ and $u_{n}=\tilde{S}\left(q_{n}\right)$. Then there exists $\tilde{u} \in \mathrm{H}_{0}^{1}(\hat{\Omega})$ with

$$
\tilde{u}_{n} \rightarrow \tilde{u} \text { in } \mathrm{H}_{0}^{1}(\hat{\Omega}) \text { for } n \rightarrow \infty,
$$

such that $u=\left.\tilde{u}\right|_{\Omega_{q}}$ holds with $u=\tilde{S}(q)$.

A direct consequence is the following existence theorem.

Theorem 1.3. Problem (1.3) has a global solution.

Proof. We have $Q^{\text {ad }} \neq \emptyset$ and $J(q, u) \geq 0$. So there exists a minimizing sequence $\left(q_{n}, u_{n}=\tilde{S}\left(q_{n}\right)\right)_{n \in \mathbb{N}}$ with

$$
j=\inf _{q \in Q^{\mathrm{ad}}} j(q)=\lim _{n \rightarrow \infty} J\left(q_{n}, u_{n}\right) .
$$

The boundedness of $Q^{\text {ad }}$ in $\mathrm{H}^{2}(I)$ implies the existence of a subsequence $\left(q_{n_{k}}\right)_{k \in \mathbb{N}} \subset\left(q_{n}\right)_{n \in \mathbb{N}}$ and $\bar{q} \in Q^{a d}$ with

$$
\begin{aligned}
& q_{n_{k}} \rightarrow \bar{q} \quad \text { in } \mathrm{H}^{2}(I), \\
& q_{n_{k}} \rightarrow \bar{q} \quad \text { in } \mathrm{C}^{0}(\bar{I}) \quad \text { for } k \rightarrow \infty,
\end{aligned}
$$


due to the compact embedding of $\mathrm{H}^{2}(I)$ into $\mathrm{C}^{0}(\bar{I})$. With Proposition 1.2 it follows that there exists $\bar{u} \in \mathrm{H}_{0}^{1}\left(\Omega_{\bar{q}}\right)$ with $\tilde{u}_{n_{k}} \rightarrow \tilde{\tilde{u}}$ in $\mathrm{H}_{0}^{1}(\hat{\Omega})$ and $\bar{u}=\tilde{S}(\bar{q})$. Now let

$$
\begin{aligned}
& J_{1}:(q, u) \mapsto\left\|u-u_{d}\right\|_{\mathrm{L}^{2}\left(\Omega_{q}\right)}^{2}=\left\|\tilde{u}-\tilde{u}_{d}\right\|_{\mathrm{L}^{2}(\hat{\Omega})}^{2}, \\
& J_{2}: q \mapsto\left\|q^{\prime \prime}\right\|_{\mathrm{L}^{2}(I)}^{2} .
\end{aligned}
$$

The functional $J_{2}$ is continuous and convex in $\mathrm{H}^{2}(I)$ and therefore weakly lower semi-continuous. By the definition of $j$ it follows that

$$
\begin{gathered}
\lim _{k \rightarrow \infty} J_{1}\left(q_{n_{k}}, u_{n_{k}}\right)=J_{1}(\bar{q}, \bar{u}), \\
\liminf _{k \rightarrow \infty} J_{2}\left(q_{n_{k}}\right) \geq J_{2}(\bar{q}) .
\end{gathered}
$$

By adding these two inequalities we get

$$
J(\bar{q}, \bar{u}) \leq \liminf _{k \rightarrow \infty} J\left(q_{n_{k}}, u_{n_{k}}\right)=j,
$$

and conclude that $J(\bar{q}, \bar{u})=j$. Hence, $(\bar{q}, \bar{u})$ is a solution of the initial problem (1.3).

Remark 1.4. Although the state equation (1.2) is linear, the solution operator $\tilde{S}$ is nonlinear and one cannot expect the reduced cost functional $j$ to be convex. Therefore uniqueness of an optimal solution cannot be shown in general.

\subsection{Optimality conditions}

In order to find the optimal solution $\bar{q}$ and to formulate optimality conditions, one usually needs some sort of derivative of the reduced cost functional $j$. In general, the derivative $j^{\prime}(q)(\delta q)$ can be represented via a domain integral. This is done later, see (3.8). Given sufficient regularity of $q$, it can also be represented via a boundary integral over the moving part of the boundary. This general principle is known as the Hadamard Formula and can be found in [26], Theorem 2.27. If $q$ is sufficiently smooth $\left(q \in \mathrm{C}^{2}(\bar{I})\right)$, then it can be shown that $j$ is Fréchet differentiable with

$$
j^{\prime}(q)(\delta q)=\int_{\Gamma_{q}}\left(\frac{1}{2}\left(u-u_{d}\right)^{2}+\partial_{n} u \partial_{n} z\right)\left\langle V_{q, \delta q}, n\right\rangle \mathrm{d} \Gamma_{q}(x)+\alpha \int_{0}^{1} q^{\prime \prime} \delta q^{\prime \prime} \mathrm{d} x .
$$

Within (1.5), the so-called adjoint variable $z \in \mathrm{H}_{0}^{1}\left(\Omega_{q}\right)$ is the weak solution of

$$
\left\{\begin{array}{rlrl}
-\Delta z+z & =u-u_{d} & & \text { in } \Omega_{q} \\
z=0 & & \text { on } \Gamma_{q}
\end{array}\right.
$$

and the vector field $V_{q, \delta q}$, describing a transformation from $\Omega_{q}$ to $\Omega_{q+\delta q}$, is given by

$$
V_{q, \delta q}(x, y)=\left(\begin{array}{c}
0 \\
(1-y) \frac{\delta q(x)}{1-q(x)}
\end{array}\right) .
$$

For an overview on how to derive such a representation we refer to [15,26]. Within Subsection 3.1 we will first present the domain integral representation of $j^{\prime}(q)(\delta q)$ which holds for all $q \in Q^{a d}$. Second, we will use that representation to show that the optimal solution $\bar{q}$ is sufficiently regular, such that (1.5) actually holds in the optimal solution $\bar{q}$.

Throughout we make the following assumption.

Assumption 1.5. We assume that for the optimal solution $\bar{q}$ under consideration the restriction $\bar{q} \leq 1-\varepsilon$ from (1.1) is not active, i.e. there exists $\delta>0$ with $\bar{q}(x) \leq 1-\varepsilon-\delta$ for all $x \in I$. 


\subsection{Transformation of the problem}

In what follows we provide an equivalent formulation of (1.3) by the transformation onto the reference domain $\Omega_{0}=(0,1)^{2}$. To this end we introduce

$$
V_{q}(x, y)=\left(\begin{array}{c}
0 \\
(1-y) q(x)
\end{array}\right)
$$

a velocity field, and let

$$
T_{q}(x, y)=\left(I d+V_{q}\right)(x, y)=\left(\begin{array}{c}
x \\
y+(1-y) q(x)
\end{array}\right)
$$

be a transformation with $\Omega_{q}=T_{q}\left(\Omega_{0}\right)$. The following functions derived from this transformation will be used in the sequel:

$$
\begin{aligned}
\mathrm{D} T_{q}(x, y) & =\left(\begin{array}{cc}
1 & 0 \\
(1-y) q^{\prime}(x) & 1-q(x)
\end{array}\right), \\
\gamma_{q}(x, y) & =\operatorname{det}\left(\mathrm{D} T_{q}(x, y)\right)=1-q(x), \\
A_{q}(x, y) & =\left(\gamma_{q} \cdot \mathrm{D} T_{q}^{-1} \cdot \mathrm{D} T_{q}^{-T}\right)(x, y)=\left(\begin{array}{cr}
1-q(x) & -(1-y) q^{\prime}(x) \\
-(1-y) q^{\prime}(x) \frac{(1-y)^{2} q^{\prime}(x)^{2}+1}{1-q(x)}
\end{array}\right), \\
A_{q, \delta q}^{\prime}(x, y) & =\left.\frac{\mathrm{d}}{\mathrm{d} t} A_{q+t \cdot \delta q}(x, y)\right|_{t=0} \\
& =\left(\begin{array}{c}
-\delta q(x) \\
-(1-y) \delta q^{\prime}(x)
\end{array}\right. \\
A_{q, \delta q, \tau q}^{\prime \prime}(x, y) & =\left.\frac{\mathrm{d} q(x)+2(1-y)^{2} q^{\prime}(x) \delta q^{\prime}(x)(1-q(x))+(1-y)^{2} q^{\prime}(x)^{2} \delta q(x)}{(1-q(x))^{2}} A_{q+t \cdot \tau q, \delta q}^{\prime}(x, y)\right|_{t=0} .
\end{aligned}
$$

With these explicit definitions at hand, one can easily derive some stability results which follow by a direct calculation and the boundedness of $Q^{\text {ad }}$ in $\mathrm{H}^{2}(I)$, as well as the fact that $u_{d}, f \in \mathrm{C}^{2,1}(\hat{\Omega})$.

Lemma 1.6. For $q, p \in Q^{\text {ad }}$ we have

- $\left\|\gamma_{q}-\gamma_{p}\right\|_{\mathrm{L}^{\infty}\left(\Omega_{0}\right)} \leq c \cdot\|q-p\|_{\mathrm{H}^{2}(I)}$,

- $\left\|T_{q}-T_{p}\right\|_{\mathrm{L}^{\infty}\left(\Omega_{0}\right)} \leq c \cdot\|q-p\|_{\mathrm{H}^{2}(I)}$,

- $\left\|T_{q}^{-1}-T_{p}^{-1}\right\|_{\mathrm{L}^{\infty}\left(\Omega_{0}\right)} \leq c \cdot\|q-p\|_{\mathrm{H}^{2}(I)}$,

- $\left\|V_{q}-V_{p}\right\|_{\mathrm{L}^{\infty}\left(\Omega_{0}\right)} \leq c \cdot\|q-p\|_{\mathrm{H}^{2}(I)}$,

- $\left\|f \circ T_{q}-f \circ T_{p}\right\|_{\mathrm{L}^{\infty}\left(\Omega_{0}\right)} \leq c \cdot\|q-p\|_{\mathrm{H}^{2}(I)}$,

- $\left\|\nabla f \circ T_{q}-\nabla f \circ T_{p}\right\|_{\mathrm{L}^{\infty}\left(\Omega_{0}\right)} \leq c \cdot\|q-p\|_{\mathrm{H}^{2}(I)}$,

- $\left\|\nabla^{2} f \circ T_{q}-\nabla^{2} f \circ T_{p}\right\|_{\mathrm{L}^{\infty}\left(\Omega_{0}\right)} \leq c \cdot\|q-p\|_{\mathrm{H}^{2}(I)}$,

- $\left\|u_{d} \circ T_{q}-u_{d} \circ T_{p}\right\|_{L^{\infty}\left(\Omega_{0}\right)} \leq c \cdot\|q-p\|_{\mathrm{H}^{2}(I)}$,

- $\left\|\nabla u_{d} \circ T_{q}-\nabla u_{d} \circ T_{p}\right\|_{\mathrm{L}^{\infty}\left(\Omega_{0}\right)} \leq c \cdot\|q-p\|_{\mathrm{H}^{2}(I)}$,

- $\left\|\nabla^{2} u_{d} \circ T_{q}-\nabla^{2} u_{d} \circ T_{p}\right\|_{L^{\infty}\left(\Omega_{0}\right)} \leq c \cdot\|q-p\|_{\mathrm{H}^{2}(I)}$,

- $\left\|\operatorname{div}\left(V_{q}\right)\right\|_{\mathrm{L}^{\infty}\left(\Omega_{0}\right)} \leq c \cdot\|q\|_{\mathrm{H}^{1}(I)}$.

Furthermore, the expressions $\gamma_{q}, T_{q}, T_{q}^{-1}, V_{q}, f \circ T_{q}, \nabla f \circ T_{q} . \nabla^{2} f \circ T_{q}, u_{d} \circ T_{q}, \nabla u_{d} \circ T_{q}, \nabla^{2} u_{d} \circ T_{q}$ and $\operatorname{div}\left(V_{q}\right)$ are bounded in $\mathrm{L}^{\infty}\left(\Omega_{0}\right)$. 
Lemma 1.7. For $q, p \in Q^{a d}, \delta q \in Q$, it holds that:

- $\left\|A_{q}\right\|_{\mathrm{L}^{\infty}\left(\Omega_{0}\right)} \leq c$,

- $\left\|A_{q}-A_{p}\right\|_{\mathrm{L}^{\infty}\left(\Omega_{0}\right)} \leq c \cdot\|q-p\|_{\mathrm{H}^{2}(I)}$,

- $\left\|A_{q, \delta q}^{\prime}\right\|_{\mathrm{L}^{\infty}\left(\Omega_{0}\right)} \leq c \cdot\|\delta q\|_{\mathrm{H}^{2}(I)}$,

- $\left\|A_{q, \delta q, \delta q}^{\prime \prime}\right\|_{\mathrm{L}^{\infty}\left(\Omega_{0}\right)} \leq c \cdot\|\delta q\|_{\mathrm{H}^{2}(I)}^{2}$.

Using transformation of integrals we obtain the following equivalence of the weak formulations on $\Omega_{q}$ and $\Omega_{0}$ :

Lemma 1.8. Let $q \in Q^{a d}, u \in \mathrm{H}_{0}^{1}\left(\Omega_{q}\right)$ and $u^{q}=u \circ T_{q} \in \mathrm{H}_{0}^{1}\left(\Omega_{0}\right)$. Then the following variational formulations are equivalent

$$
\begin{array}{ccc}
\int_{\Omega_{q}}(\nabla u \nabla z+u z) \mathrm{d} x=\int_{\Omega_{q}} f z \mathrm{~d} x & \forall z \in \mathrm{H}_{0}^{1}\left(\Omega_{q}\right), \\
\int_{\Omega_{0}}\left(\nabla u^{q} A_{q} \nabla z^{q}+u^{q} z^{q} \gamma_{q}\right) \mathrm{d} x=\int_{\Omega_{0}}\left(f \circ T_{q}\right) z^{q} \gamma_{q} \mathrm{~d} x & \forall z^{q} \in \mathrm{H}_{0}^{1}\left(\Omega_{0}\right) .
\end{array}
$$

The advantage of (1.14) over (1.13) is its independence of the domain, instead the coefficients of the matrix $A_{q}$ are changeable. To shorten notation, we will also from now on make use of the following forms

$$
\begin{aligned}
a(q)(u, z) & =\int_{\Omega_{0}}\left(\nabla u A_{q} \nabla z+u z \gamma_{q}\right) \mathrm{d} x, \\
l(q)(z) & =\int_{\Omega_{0}}\left(f \circ T_{q}\right) z \gamma_{q} \mathrm{~d} x .
\end{aligned}
$$

Then (1.14) can be written as

$$
a(q)\left(u^{q}, z^{q}\right)=l(q)\left(z^{q}\right) \quad \forall z^{q} \in \mathrm{H}_{0}^{1}\left(\Omega_{0}\right) .
$$

Lemma 1.8 motivates the introduction of another solution operator $S$ which assigns to each control $q$ the "transported" solution, i.e. let $S(q)=\tilde{S}(q) \circ T_{q} \in \mathrm{H}_{0}^{1}\left(\Omega_{0}\right)$.

In the following lemmata we summarize some properties of the bilinear form $a(q)(\cdot, \cdot)$ and the linear functional $l(q)(\cdot)$, which are direct consequences of the definitions, Lemma 1.6 and Lemma 1.7.

Lemma 1.9. The bilinear form $a(q)(\cdot, \cdot)$ is continuous and coercive in $\mathrm{H}_{0}^{1}\left(\Omega_{0}\right)$, i.e. there exist $c_{1}, c_{2}>0$, independent of $q$, such that for all $q \in Q^{a d}$ and $u, z \in \mathrm{H}_{0}^{1}\left(\Omega_{0}\right)$ it holds that

$$
\begin{aligned}
& |a(q)(u, z)| \leq c_{1} \cdot\|\nabla u\|_{\mathrm{L}^{2}\left(\Omega_{0}\right)} \cdot\|\nabla z\|_{\mathrm{L}^{2}\left(\Omega_{0}\right)}, \\
& a(q)(u, u) \geq c_{2} \cdot\|\nabla u\|_{\mathrm{L}^{2}\left(\Omega_{0}\right)}^{2} .
\end{aligned}
$$

Furthermore, there exists $c_{3}>0$, independent of $q$, such that for $u \in \mathrm{W}^{1, p}\left(\Omega_{0}\right), p \in[1, \infty]$ and $z \in \mathrm{W}^{1, p^{\prime}}\left(\Omega_{0}\right)$ with $p^{-1}+p^{\prime-1}=1$ the following Hölder-like inequality holds

$$
|a(q)(u, z)| \leq c_{3} \cdot\|u\|_{\mathrm{W}^{1, p}\left(\Omega_{0}\right)} \cdot\|z\|_{\mathrm{W}^{1, p^{\prime}}\left(\Omega_{0}\right)} .
$$

Lemma 1.10. For $q, p \in Q^{a d}$ and $u, z \in \mathrm{H}^{1}\left(\Omega_{0}\right)$ it holds that

$$
|a(q)(u, z)-a(p)(u, z)| \leq c \cdot\|q-p\|_{\mathrm{H}^{2}(I)} \cdot\|u\|_{\mathrm{H}^{1}\left(\Omega_{0}\right)} \cdot\|z\|_{\mathrm{H}^{1}\left(\Omega_{0}\right)} .
$$

Lemma 1.11. For $q, p \in Q^{a d}$ and $z \in \mathrm{H}^{1}\left(\Omega_{0}\right)$ it holds that

$$
|l(q)(z)-l(p)(z)| \leq c \cdot\|q-p\|_{\mathrm{H}^{2}(I)} \cdot\|z\|_{\mathrm{L}^{2}\left(\Omega_{0}\right)} .
$$


In the following sections we will make heavy use of various regularity results for elliptic partial differential equations. Here we state them all at once.

Theorem 1.12. Let $\Omega \subset \mathbb{R}^{2}$ be bounded with Lipschitz boundary $\Gamma, f \in \mathrm{H}^{-1}(\Omega)$, and let the matrix $A$ be symmetric and uniformly elliptic with coefficients $a_{i, j} \in \mathrm{L}^{\infty}\left(\Omega_{0}\right)$. Furthermore, let $u \in \mathrm{H}_{0}^{1}(\Omega)$ be the weak solution of

$$
\left\{\begin{aligned}
-\operatorname{div}(A \nabla u)=f & \text { in } \Omega, \\
u=0 & \text { on } \Gamma .
\end{aligned}\right.
$$

1. If $1 / 2 \geq t>s>0$ and the coefficients of $A$ belong to $\mathrm{C}^{0, t}(\bar{\Omega})$, then for all $f \in \mathrm{H}^{-1+s}(\Omega)$ it holds $u \in \mathrm{H}_{0}^{1+s}(\Omega)$ and there exists $c_{s}>0$ with $\|u\|_{\mathrm{H}^{1+s}(\Omega)} \leq c_{s} \cdot\|f\|_{\mathrm{H}^{-1+s}(\Omega)}$.

2. If the coefficients of $A$ are Lipschitz and there exists $\varepsilon>0$ with $f \in \mathrm{H}^{-1 / 2+\varepsilon}(\Omega)$, then $u \in \mathrm{H}^{3 / 2}(\Omega)$. In addition, if $f \in \mathrm{L}^{2}(\Omega)$, then there exists $c>0$ with $\|u\|_{\mathrm{H}^{3 / 2}(\Omega)} \leq c \cdot\|f\|_{\mathrm{L}^{2}(\Omega)}$.

3. If $\Omega$ is polygonal and convex, the coefficients of $A$ are Lipschitz and $f \in \mathrm{L}^{2}(\Omega)$, then $u \in \mathrm{H}^{2}(\Omega)$ and there exists $c>0$, depending only on the diameter of $\Omega$, with $\|u\|_{\mathrm{H}^{2}(\Omega)} \leq c \cdot\|f\|_{\mathrm{L}^{2}(\Omega)}$.

4. If $\Omega$ is polygonal and convex, and the coefficients of $A$ are Lipschitz then there exists $p_{\Omega}>2$ such that for all $p<p_{\Omega}$ and $f \in \mathrm{L}^{p}(\Omega)$ it holds that $u \in \mathrm{W}^{2, p}(\Omega)$ and there exists $c>0$, depending on the Lipschitz-constant of $A$, such that $\|u\|_{\mathrm{W}^{2, p}(\Omega)} \leq c \cdot\|f\|_{\mathrm{L}^{p}(\Omega)}$.

Proof. Part (1) can be found in [22], (2) can be found in [16,17,24], part (3) is proven in $[12,18]$ and the proof for the last part (4) can be found in [12].

Remark 1.13. All the statements of Theorem 1.12 remain true if a zero order term pops up. Let $b \in \mathrm{C}^{1}(\bar{\Omega})$ with $b \geq c>0$ in $\Omega$. Let now $u \in \mathrm{H}_{0}^{1}(\Omega)$ be the weak solution of

$$
\left\{\begin{aligned}
-\operatorname{div}(A \nabla u)+b u & =f & & \text { in } \Omega, \\
u & =0 & & \text { on } \Gamma .
\end{aligned}\right.
$$

Which can equivalently be rewritten as

$$
\left\{\begin{aligned}
-\operatorname{div}(A \nabla u) & =f-b u \text { in } \Omega, \\
u & =0 \text { on } \Gamma .
\end{aligned}\right.
$$

Now we can apply Theorem 1.12 to (1.17) and use the common $\mathrm{H}^{1}$-regularity results to show that Theorem 1.12 still holds.

Corollary 1.14. For $q \in Q^{\text {ad }}$ it holds that the corresponding state $u$ as well as the corresponding adjoint state $z$, defined via (1.2) and (1.6), respectively, possess the higher regularity $u, z \in \mathrm{H}^{3 / 2}\left(\Omega_{q}\right)$.

Proof. This corollary follows from Theorem 1.12, part (2), together with Remark 1.13.

\section{Discretization}

In order to solve (1.3) numerically we discretize the problem using (bi)linear finite elements. To this end, we first discretize the control $q$, afterwards the state $u$ is being discretized. For the discretization of the control we split the interval $(0,1)$ into $N$ pairwise disjoint nonempty subintervals, i.e. we choose $0=x_{0}<x_{1}<\ldots<$ $x_{N-1}<x_{N}=1$ and set $I_{i}=\left(x_{i-1}, x_{i}\right)$ for $i \in\{1, \ldots, N\}$. Furthermore, let $\left|I_{i}\right| \leq \sigma$ for a given discretization parameter $\sigma>0$ and all $i \in\{1, \ldots, N\}$. We use Hermite elements to define the set of the discretized controls by

$$
\begin{aligned}
Q_{\sigma} & =\left\{q \in Q|q|_{I_{i}} \in \mathcal{P}_{3}\left(I_{i}\right) \forall i \in\{1, \ldots, N\}\right\}, \\
Q_{\sigma}^{\mathrm{ad}} & =Q^{\text {ad }} \cap Q_{\sigma},
\end{aligned}
$$


where $\mathcal{P}_{n}(J)$ shall denote the set of all polynomials of degree less than or equal to $n$ over the set $J$. It can be seen immediately that for $q_{\sigma} \in Q_{\sigma}^{\text {ad }}$ we have $q_{\sigma} \in \mathrm{W}^{2, \infty}(I) \hookrightarrow \mathrm{C}^{1,1}(\bar{I})$. As polynomials of degree 3 have 4 degrees of freedom, any $q_{\sigma} \in Q_{\sigma}^{a d}$ is uniquely determined by the values of $q_{\sigma}\left(x_{i}\right)$ and $q_{\sigma}^{\prime}\left(x_{i}\right)$. As $Q_{\sigma}^{a d} \subset \mathrm{H}_{0}^{1}(I)$, the function values at $x_{0}$ and $x_{N}$ are set to 0 .

Corollary 2.1. For $q_{\sigma} \in Q_{\sigma}^{a d}$ we have $S\left(q_{\sigma}\right) \in \mathrm{H}^{2}\left(\Omega_{0}\right)$ and there exists $c>0$ independent of $\sigma$ with $\left\|S\left(q_{\sigma}\right)\right\|_{\mathrm{H}^{2}\left(\Omega_{0}\right)} \leq c \cdot\left\|f \circ T_{q_{\sigma}} \gamma_{q_{\sigma}}\right\|_{\mathrm{L}^{2}\left(\Omega_{0}\right)}$.

Proof. Because of $q_{\sigma} \in \mathrm{C}^{1,1}(\bar{I})$ the matrix $A_{q_{\sigma}}$ is Lipschitz, and the proof follows with Theorem 1.12, part (3).

The problem with discretized control now reads as

$$
\text { Minimize } j\left(q_{\sigma}\right), \quad q_{\sigma} \in Q_{\sigma}^{a d} .
$$

Now we discretize the state $u$. As later on all computations are carried out on the reference domain $\Omega_{0}$, we just discretize the "transported" solution $u^{q}$. Let $\left(\mathcal{T}_{h}\right)_{h>0}$ be a family of partitions of $\Omega_{0}$ into either triangles or quadrilaterals which should also fulfill the usual regularity assumptions like shape-regularity etc. $h_{K}$ shall denote the diameter of the element $K$ and $h=\max _{K \in \mathcal{T}_{h}} h_{K}$ is a discretization parameter for the state. The space of discrete test functions is now defined as

$$
\begin{aligned}
& V_{h}=\left\{v_{h} \in \mathrm{H}_{0}^{1}\left(\Omega_{0}\right)\left|v_{h}\right|_{K} \in \mathcal{P}_{1}(K) \forall K \in \mathcal{T}_{h}\right\} \text { for triangles, and } \\
& V_{h}=\left\{v_{h} \in \mathrm{H}_{0}^{1}\left(\Omega_{0}\right)\left|v_{h}\right|_{K} \in \mathcal{Q}_{1}(K) \forall K \in \mathcal{T}_{h}\right\} \text { for quadrilaterals. }
\end{aligned}
$$

Here $\mathcal{Q}_{n}(K)$ shall denote the set of all polynomials over $K$ whose exponents in $x$ and $y$ are less than or equal to $n$. We are now able to formulate the fully discretized problem

$$
\text { Minimize } J\left(q_{\sigma}, u^{h} \circ T_{q_{\sigma}}^{-1}\right)=\frac{1}{2}\left\|u^{h} \circ T_{q_{\sigma}}^{-1}-u_{d}\right\|_{\mathrm{L}^{2}\left(\Omega_{q_{\sigma}}\right)}^{2}+\frac{\alpha}{2}\left\|q_{\sigma}^{\prime \prime}\right\|_{\mathrm{L}^{2}(I)}^{2}, \quad q_{\sigma} \in Q_{\sigma}^{a d}, u^{h} \in V_{h},
$$

where $q_{\sigma}$ and $u^{h}$ fulfill the following variational equality

$$
a\left(q_{\sigma}\right)\left(u^{h}, z^{h}\right)=l\left(q_{\sigma}\right)\left(z^{h}\right) \quad \forall z^{h} \in V_{h},
$$

which is just the discrete counterpart of (1.14).

Again, for each $q \in Q^{a d}$ there exists a unique $u^{h}(q) \in V_{h}$ which solves (2.5). Therefore we can define a discrete analogue to the operator $S$ and the functional $j$.

Definition 2.2. Let $S_{h}: Q^{a d} \mapsto V_{h}$ be the operator which assigns to each $q \in Q^{a d}$ the unique solution $u^{h}$ of $(2.5)$, and let $j_{h}: Q^{a d} \mapsto \mathbb{R}, j_{h}(q)=J\left(q, S_{h}(q) \circ T_{q}^{-1}\right)$ be the reduced discrete cost functional.

Next, one is interested whether the problems (2.3) and (2.4) also have a solution, and what happens if we take the limit for $(\sigma, h) \rightarrow 0$. Again, most of the proofs are similar to [13] and can easily be transformed to our problem. We will just list two of the most important theorems which answer the questions from above.

Theorem 2.3. The (partially) discretized problems (2.3) and (2.4) have a solution.

Theorem 2.4. Let $\left(\bar{q}_{\sigma_{n}, h_{n}}, \bar{u}^{\sigma_{n}, h_{n}}=S_{h}\left(\bar{q}_{\sigma_{n}, h_{n}}\right)\right)_{n \in \mathbb{N}}$ be a sequence of optimal pairs for (2.4) with $\left(\sigma_{n}, h_{n}\right) \rightarrow$ 0 for $n \rightarrow \infty$. Then there exists $\bar{q} \in Q^{a d}$ and $\bar{u} \in \mathrm{H}_{0}^{1}\left(\Omega_{\bar{q}}\right)$ with $\bar{u}=\tilde{S}(\bar{q})$ and a subsequence $\left(\bar{q}_{\sigma_{n_{k}}, h_{n_{k}}}, \bar{u}^{\sigma_{n_{k}}, h_{n_{k}}}\right)_{k \in \mathbb{N}} \subset\left(\bar{q}_{\sigma_{n}, h_{n}}, \bar{u}^{\sigma_{n}, h_{n}}\right)_{n \in \mathbb{N}}$ with

$$
\begin{array}{lll}
\bar{q}_{\sigma_{n_{k}}, h_{n_{k}}} \rightarrow \bar{q} & & \text { in } \mathrm{H}^{2}(I), \\
\bar{u}^{\sigma_{n_{k}}, h_{n_{k}}} \rightarrow \bar{u} \circ T_{\bar{q}} & & \text { in } \mathrm{H}_{0}^{1}\left(\Omega_{0}\right)
\end{array}
$$

for $k \rightarrow \infty$,

and the pair $(\bar{q}, \bar{u})$ is a solution of (1.3).

Remark 2.5. Theorem 2.4 also holds if a sequence $\left(\bar{q}_{\sigma, h}, \bar{u}_{\sigma, h}\right)_{\sigma, h>0}$ of local optimal pairs for (2.4) is considered. In that case, the limit $(\bar{q}, \bar{u})$ is of course just a local solution of (1.3). 


\section{A PRIORI ERROR ESTIMATES}

Within this section we are going to prove some a priori bounds for the error between the optimal control $\bar{q}$ in the continuous case and its fully discretized counterpart $\bar{q}_{\sigma, h}$. As the optimal control may not be unique, it is not guaranteed that one can find converging sequences of globally optimal discretized solutions for each $\bar{q}$. However, we will show that for each $\bar{q}$ one can find converging subsequences of at least local optimal solutions of $(2.4)$.

Let $\bar{q}$ be a local solution of (1.3) which satisfies Assumption 1.5. The optimality conditions of first and second order, respectively, are

$$
\begin{aligned}
j^{\prime}(\bar{q})(\delta q) & =0 \quad \forall \delta q \in Q, \\
j^{\prime \prime}(\bar{q})(\delta q, \delta q) \geq 0 & \forall \delta q \in Q .
\end{aligned}
$$

The differentiability of the reduced cost functional $j$ will be shown within this section.

In order to proof our main result later on, we have to make the following assumption, which is just slightly stronger than (3.2).

Assumption 3.1. Let $\bar{q}$ be a local minimum of (1.3). We assume that

$$
j^{\prime \prime}(\bar{q})(\delta q, \delta q)>0 \quad \forall \delta q \in Q \backslash\{0\} .
$$

Now we are able to state our main result:

Theorem 3.2. Let $\bar{q}$ be a local solution of (1.3) which fulfills the Assumptions 1.5 and 3.1. Then, for $(\sigma, h)$ sufficiently small, there exist $c_{1}, c_{2}>0$, independent of $\sigma$ and $h$, with

$$
\left\|\bar{q}-\bar{q}_{\sigma, h}\right\|_{\mathrm{H}^{2}(I)} \leq c_{1} \cdot \sigma^{2}+c_{2} \cdot h^{2},
$$

where $\left(\bar{q}_{\sigma, h}\right)_{\sigma, h>0}$ is a converging sequence of local optimal solutions of (2.4).

In order to prove Theorem 3.2, the error will be split,

$$
\left\|\bar{q}-\bar{q}_{\sigma, h}\right\|_{\mathrm{H}^{2}(I)} \leq\left\|\bar{q}-\bar{q}_{\sigma}\right\|_{\mathrm{H}^{2}(I)}+\left\|\bar{q}_{\sigma}-\bar{q}_{\sigma, h}\right\|_{\mathrm{H}^{2}(I)},
$$

where $\left(\bar{q}_{\sigma}\right)_{\sigma>0}$ and $\left(\bar{q}_{\sigma, h}\right)_{\sigma, h>0}$ are sequences of local optimal solutions of (2.3) and (2.4), respectively, which converge to $\bar{q}$ in $\mathrm{H}^{2}(I)$. The existence of such sequences will be shown in the sequel.

The first part on the right hand side of (3.3) refers to the discretization of the control, whereas the latter part refers to the discretization of the state. These two parts will be estimated separately.

In addition, from now on let $\bar{q}$ be a fixed local solution of (1.3) which fulfills Assumption 1.5 and 3.1.

First of all, we have to show that the operator $S$ is at least twice Fréchet differentiable. This can be done by using the Implicit Function Theorem for Banach spaces. The following version can be found in [4], Theorem 2.3.

Theorem 3.3. Let $F \in \mathrm{C}^{k}\left(X^{a d} \times Y, Z\right), k \geq 1$, where $Y$ and $Z$ are Banach spaces and $X^{\text {ad }}$ is an open subset of Banach space $X$. Suppose that $F\left(x^{*}, y^{*}\right)=0$ and that $F_{y}^{\prime}\left(x^{*}, y^{*}\right)$ is continuously invertible. Then there exist neighbourhoods $\Theta$ of $x^{*}$ in $X, \Phi$ of $y^{*}$ in $Y$ and a map $g \in \mathrm{C}^{k}(\Theta, Y)$ such that $F(x, g(y))=0$ for all $x \in \Theta$. Furthermore, $F(x, y)=0$ for $(x, y) \in \Theta \times \Phi$ implies $y=g(x)$.

Corollary 3.4. The Operator $S$ is at least twice continuously Fréchet differentiable.

Proof. We set $X^{a d}=\operatorname{int}\left(Q^{a d}\right), X=Q, Y=\mathrm{H}_{0}^{1}\left(\Omega_{0}\right)$ and $Z=\mathrm{H}^{-1}\left(\Omega_{0}\right)$. Furthermore, define $F: Q^{a d} \times$ $\mathrm{H}_{0}^{1}\left(\Omega_{0}\right) \mapsto \mathrm{H}^{-1}\left(\Omega_{0}\right), F(q, u)=a(q)(u, \cdot)-l(q)(\cdot)$. Then $F$ is affine in $u$ and twice continuously differentiable with respect to $q$, as can be shown by a direct computation using the definitions (1.8)-(1.12). The result then follows with Theorem 3.3. 
We now recall the definition of the operator $S$ and its derivatives, which follow by a direct calculation.

1. $u=S(q) \in \mathrm{H}_{0}^{1}\left(\Omega_{0}\right)$ is the solution of

$$
a(q)(u, z)=\left(f \circ T_{q}, z \gamma_{q}\right) \quad \forall z \in \mathrm{H}_{0}^{1}\left(\Omega_{0}\right) .
$$

2. $\delta u=S^{\prime}(q)(\delta q) \in \mathrm{H}_{0}^{1}\left(\Omega_{0}\right)$ is the solution of

$$
\begin{aligned}
a(q)(\delta u, z)= & \left(\left(\nabla f \circ T_{q}\right)^{T} \cdot V_{\delta q}, z \gamma_{q}\right)+\left(f \circ T_{q}, z \operatorname{div}\left(V_{\delta q}\right)\right)-\left(\nabla u, A_{q, \delta q}^{\prime} \nabla z\right) \\
& -\left(u, z \operatorname{div}\left(V_{\delta q}\right)\right) \quad \forall z \in \mathrm{H}_{0}^{1}\left(\Omega_{0}\right) .
\end{aligned}
$$

3. $\delta \tau u=S^{\prime \prime}(q)(\delta q, \tau q) \in \mathrm{H}_{0}^{1}\left(\Omega_{0}\right)$ is the solution of

$$
\begin{aligned}
a(q)(\delta \tau u, z)= & \left(V_{\tau q}^{T} \cdot \nabla^{2} f \circ T_{q} \cdot V_{\delta q}, z \gamma_{q}\right)-\left(\nabla u, A_{q, \delta q, \tau q}^{\prime \prime} \nabla z\right) \\
& +\left(\left(\nabla f \circ T_{q}\right)^{T} \cdot V_{\tau q}, z \operatorname{div}\left(V_{\delta q}\right)\right)+\left(\left(\nabla f \circ T_{q}\right)^{T} \cdot V_{\delta q}, z \operatorname{div}\left(V_{\tau q}\right)\right) \\
& -\left(\nabla \tau u, A_{q, \delta q}^{\prime} \nabla z\right)-\left(\nabla \delta u, A_{q, \tau q}^{\prime} \nabla z\right) \\
& -\left(\tau u, z \operatorname{div}\left(V_{\delta q}\right)\right)-\left(\delta u, z \operatorname{div}\left(V_{\tau q}\right)\right) \quad \forall z \in \mathrm{H}_{0}^{1}\left(\Omega_{0}\right),
\end{aligned}
$$

where $\tau u=S^{\prime}(q)(\tau q)$.

Remark 3.5. The representations (3.6) as well as (3.9) show that the second derivatives are symmetric with respect to the directions.

Lemma 3.6. For $q \in Q^{a d}, \delta q \in Q$ it holds with $q$-independent constants that

1. $\|S(q)\|_{\mathrm{H}^{1}\left(\Omega_{0}\right)} \leq c$,

2. $\left\|S^{\prime}(q)(\delta q)\right\|_{\mathrm{H}^{1}\left(\Omega_{0}\right)} \leq c \cdot\|\delta q\|_{\mathrm{H}^{2}(I)}$,

3. $\left\|S^{\prime \prime}(q)(\delta q, \delta q)\right\|_{\mathrm{H}^{1}\left(\Omega_{0}\right)} \leq c \cdot\|\delta q\|_{\mathrm{H}^{2}(I)}^{2}$.

Proof. (1) By the uniform coercivity of $a(q)(\cdot, \cdot)$ we have

$$
\begin{aligned}
c \cdot\|S(q)\|_{\mathrm{H}^{1}\left(\Omega_{0}\right)}^{2} & \leq a(q)(S(q), S(q))=l(q)(S(q)) \\
& \leq\left\|\gamma_{q}\right\|_{\mathrm{L}^{\infty}\left(\Omega_{0}\right)} \cdot\left\|f \circ T_{q}\right\|_{\mathrm{L}^{2}\left(\Omega_{0}\right)} \cdot\|S(q)\|_{\mathrm{H}^{1}\left(\Omega_{0}\right)} \leq c \cdot\|S(q)\|_{\mathrm{H}^{1}\left(\Omega_{0}\right)} .
\end{aligned}
$$

(2) and (3) are also proven using the uniform coercivity of $a(q)(\cdot, \cdot)$ as well as the Lemmata 1.6 and 1.7.

Lemma 3.7. For $q, p \in Q^{a d}, \delta q \in Q$ it holds that

1. $\|S(q)-S(p)\|_{\mathrm{H}^{1}\left(\Omega_{0}\right)} \leq c \cdot\|q-p\|_{\mathrm{H}^{2}(I)}$,

2. $\left\|S^{\prime}(q)(\delta q)-S^{\prime}(p)(\delta q)\right\|_{\mathrm{H}^{1}\left(\Omega_{0}\right)} \leq c \cdot\|q-p\|_{\mathrm{H}^{2}(I)} \cdot\|\delta q\|_{\mathrm{H}^{2}(I)}$,

3. $\left\|S^{\prime \prime}(q)(\delta q, \delta q)-S^{\prime \prime}(p)(\delta q, \delta q)\right\|_{\mathrm{H}^{1}\left(\Omega_{0}\right)} \leq c \cdot\|q-p\|_{\mathrm{H}^{2}((0,1))} \cdot\|\delta q\|_{\mathrm{H}^{2}(I)}^{2}$.

Proof. (1) Let $e=S(q)-S(p)$. With Lemma 1.10 we have

$$
\begin{aligned}
c \cdot\|e\|_{\mathrm{H}^{1}\left(\Omega_{0}\right)}^{2} & \leq a(q)(e, e)=a(q)(S(q), e)-a(q)(S(p), e) \\
& \leq a(q)(S(q), e)-a(p)(S(p), e)+c \cdot\|S(p)\|_{\mathrm{H}^{1}\left(\Omega_{0}\right)} \cdot\|e\|_{\mathrm{H}^{1}\left(\Omega_{0}\right)} \cdot\|q-p\|_{\mathrm{H}^{2}(I)} .
\end{aligned}
$$

Due to the definition of $S(q), S(p)$ and Lemma 1.11 we get

$$
a(q)(S(q), e)-a(p)(S(p), e)=l(q)(e)-l(p)(e) \leq c \cdot\|q-p\|_{\mathrm{H}^{2}(I)} \cdot\|e\|_{\mathrm{H}^{1}\left(\Omega_{0}\right)} .
$$

As $\|S(p)\|_{\mathrm{H}^{1}\left(\Omega_{0}\right)}$ is bounded, the proof for this part is finished.

(2) and (3) are proven similarly to the first part, one additionally has to make use of the Lemmata 1.6, 1.10, 1.11 and 3.6. 
In what follows we will also need some stability results concerning the reduced cost functional $j$ and its derivatives. The fact that $j$ is at least twice differentiable follows from Corollary 3.4. A direct calculation yields

$$
\begin{aligned}
j(q)= & \frac{1}{2}\left(S(q)-u_{d} \circ T_{q},\left(S(q)-u_{d} \circ T_{q}\right) \gamma_{q}\right)+\frac{\alpha}{2}\left(q^{\prime \prime}, q^{\prime \prime}\right)_{\mathrm{L}^{2}(I)}, \\
j^{\prime}(q)(\delta q)= & \frac{1}{2}\left(S(q)-u_{d} \circ T_{q},\left(S(q)-u_{d} \circ T_{q}\right) \operatorname{div}\left(V_{\delta q}\right)\right) \\
& +\left(S^{\prime}(q)(\delta q)-\left(\nabla u_{d} \circ T_{q}\right)^{T} \cdot V_{\delta q},\left(S(q)-u_{d} \circ T_{q}\right) \gamma_{q}\right)+\alpha\left(q^{\prime \prime}, \delta q^{\prime \prime}\right)_{\mathrm{L}^{2}(I)}, \\
j^{\prime \prime}(q)(\delta q, \tau q)= & \left(S^{\prime}(q)(\delta q)-\left(\nabla u_{d} \circ T_{q}\right)^{T} \cdot V_{\delta q},\left(S^{\prime}(q)(\tau q)-\left(\nabla u_{d} \circ T_{q}\right)^{T} \cdot V_{\tau q}\right) \gamma_{q}\right) \\
& +\left(S(q)-u_{d} \circ T_{q},\left(S^{\prime}(q)(\delta q)-\left(\nabla u_{d} \circ T_{q}\right)^{T} \cdot V_{\delta q}\right) \operatorname{div}\left(V_{\tau q}\right)\right) \\
& +\left(S(q)-u_{d} \circ T_{q},\left(S^{\prime}(q)(\tau q)-\left(\nabla u_{d} \circ T_{q}\right)^{T} \cdot V_{\tau q}\right) \operatorname{div}\left(V_{\delta q}\right)\right) \\
& +\left(S(q)-u_{d} \circ T_{q},\left(S^{\prime \prime}(q)(\delta q, \tau q)-V_{\tau q}^{T} \cdot \nabla^{2} u_{d} \circ T_{q} \cdot V_{\delta q}\right) \gamma_{q}\right)+\alpha\left(\delta q^{\prime \prime}, \tau q^{\prime \prime}\right)_{\mathrm{L}^{2}(I)} .
\end{aligned}
$$

Lemma 3.8. For $q, p \in Q^{a d}, \delta q \in Q$ it holds that

1. $|j(q)-j(p)| \leq c \cdot\|q-p\|_{\mathrm{H}^{2}(I)}$,

2. $\left|j^{\prime}(q)(\delta q)-j^{\prime}(p)(\delta q)\right| \leq c \cdot\|q-p\|_{\mathrm{H}^{2}(I)} \cdot\|\delta q\|_{\mathrm{H}^{2}(I)}$,

3. $\left|j^{\prime \prime}(q)(\delta q, \delta q)-j^{\prime \prime}(p)(\delta q, \delta q)\right| \leq c \cdot\|q-p\|_{\mathrm{H}^{2}(I)} \cdot\|\delta q\|_{\mathrm{H}^{2}(I)}^{2}$.

Proof. (1) With the inequality of Cauchy-Schwarz and the definition of $Q^{\text {ad }}$ we get

$$
\left(q^{\prime \prime}, q^{\prime \prime}\right)_{\mathrm{L}^{2}(I)}-\left(p^{\prime \prime}, p^{\prime \prime}\right)_{\mathrm{L}^{2}(I)}=\left(q^{\prime \prime}-p^{\prime \prime}, q^{\prime \prime}+p^{\prime \prime}\right)_{\mathrm{L}^{2}(I)} \leq c \cdot\|q-p\|_{\mathrm{H}^{2}(I)} .
$$

Applying the Lemmata 1.6, 3.6, 3.7 and again Cauchy-Schwarz's inequality one gets

$$
\begin{aligned}
& \left(S(q)-u_{d} \circ T_{q},\left(S(q)-u_{d} \circ T_{q}\right) \gamma_{q}\right)-\left(S(p)-u_{d} \circ T_{p},\left(S(p)-u_{d} \circ T_{p}\right) \gamma_{p}\right) \\
& =\left(S(q)-u_{d} \circ T_{q}-S(p)+u_{d} \circ T_{p},\left(S(q)-u_{d} \circ T_{q}+S(p)-u_{d} \circ T_{p}\right) \gamma_{q}\right)+\left(\left(S(p)-u_{d} \circ T_{p}\right)^{2}, \gamma_{q}-\gamma_{p}\right) \\
& \leq c \cdot\left(\|S(q)-S(p)\|_{\mathrm{L}^{2}\left(\Omega_{0}\right)}+\left\|u_{d} \circ T_{q}-u_{d} \circ T_{p}\right\|_{\mathrm{L}^{2}\left(\Omega_{0}\right)}\right)+c \cdot\left\|\gamma_{q}-\gamma_{p}\right\|_{\mathrm{L}^{2}\left(\Omega_{0}\right)} \\
& \leq c \cdot\|q-p\|_{\mathrm{H}^{2}(I)} .
\end{aligned}
$$

(2) First of all, we have

$$
\left(q^{\prime \prime}, \delta q^{\prime \prime}\right)_{\mathrm{L}^{2}(I)}-\left(p^{\prime \prime}, \delta q^{\prime \prime}\right)_{\mathrm{L}^{2}(I)}=\left(q^{\prime \prime}-p^{\prime \prime}, \delta q^{\prime \prime}\right)_{\mathrm{L}^{2}(I)} \leq\|q-p\|_{\mathrm{H}^{2}(I)} \cdot\|\delta q\|_{\mathrm{H}^{2}(I)}
$$

Furthermore

$\left(S(q)-u_{d} \circ T_{q},\left(S(q)-u_{d} \circ T_{q}\right) \operatorname{div}\left(V_{\delta q}\right)\right)-\left(S(p)-u_{d} \circ T_{p},\left(S(p)-u_{d} \circ T_{p}\right) \operatorname{div}\left(V_{\delta q}\right)\right) \leq c \cdot\|q-p\|_{\mathrm{H}^{2}(I)} \cdot\|\delta q\|_{\mathrm{H}^{2}(I)}$ as we can apply the same steps as in the proof of the first part and also use Lemma 1.6. At last

$$
\begin{aligned}
& \left(S^{\prime}(q)(\delta q)-\left(\nabla u_{d} \circ T_{q}\right)^{T} \cdot V_{\delta q},\left(S(q)-u_{d} \circ T_{q}\right) \gamma_{q}\right)-\left(S^{\prime}(p)(\delta q)-\left(\nabla u_{d} \circ T_{p}\right)^{T} \cdot V_{\delta q},\left(S(p)-u_{d} \circ T_{p}\right) \gamma_{p}\right) \\
= & \left(S^{\prime}(q)(\delta q)-\left(\nabla u_{d} \circ T_{q}\right)^{T} \cdot V_{\delta q}-S^{\prime}(p)(\delta q)+\left(\nabla u_{d} \circ T_{p}\right)^{T} \cdot V_{\delta q},\left(S(q)-u_{d} \circ T_{q}\right) \gamma_{q}\right) \\
& +\left(S^{\prime}(p)(\delta q)-\left(\nabla u_{d} \circ T_{p}\right)^{T} \cdot V_{\delta q},\left(S(q)-u_{d} \circ T_{q}-S(p)+u_{d} \circ T_{p}\right) \gamma_{q}\right) \\
& +\left(S^{\prime}(p)(\delta q)-\left(\nabla u_{d} \circ T_{p}\right)^{T} \cdot V_{\delta q},\left(S(p)-u_{d} \circ T_{p}\right) \cdot\left(\gamma_{q}-\gamma_{p}\right)\right)
\end{aligned}
$$


again we use the Lemmas 1.6, 3.6, 3.7 and get

$$
\leq c \cdot\|q-p\|_{\mathrm{H}^{2}(I)} \cdot\|\delta q\|_{\mathrm{H}^{2}(I)}
$$

(3) All the terms which do not contain a second derivative can be estimated in the same way as done in the first two parts, thus we only have to have a look at

$$
\begin{aligned}
& \left(S(q)-u_{d} \circ T_{q},\left(S^{\prime \prime}(q)(\delta q, \delta q)-V_{\delta q}^{T} \cdot \nabla^{2} u_{d} \circ T_{q} \cdot V_{\delta q}\right) \gamma_{q}\right) \\
& -\left(S(p)-u_{d} \circ T_{p},\left(S^{\prime \prime}(p)(\delta q, \delta q)-V_{\delta q}^{T} \cdot \nabla^{2} u_{d} \circ T_{p} \cdot V_{\delta q}\right) \gamma_{p}\right) \\
= & \left(S(q)-u_{d} \circ T_{q}-S(p)+u_{d} \circ T_{p},\left(S^{\prime \prime}(q)(\delta q, \delta q)-V_{\delta q}^{T} \cdot \nabla^{2} u_{d} \circ T_{q} \cdot V_{\delta q}\right) \gamma_{q}\right) \\
& +\left(S(p)-u_{d} \circ T_{p},\left(S^{\prime \prime}(q)(\delta q, \delta q)-V_{\delta q}^{T} \cdot \nabla^{2} u_{d} \circ T_{q} \cdot V_{\delta q}-S^{\prime \prime}(p)(\delta q, \delta q)+V_{\delta q}^{T} \cdot \nabla^{2} u_{d} \circ T_{p} \cdot V_{\delta q}\right) \gamma_{q}\right) \\
& +\left(S(p)-u_{d} \circ T_{p},\left(S^{\prime \prime}(p)(\delta q, \delta q)-V_{\delta q}^{T} \cdot \nabla^{2} u_{d} \circ T_{p} \cdot V_{\delta q}\right)\left(\gamma_{q}-\gamma_{p}\right)\right) \\
\leq & c \cdot\|q-p\|_{\mathrm{H}^{2}(I)} \cdot\|\delta q\|_{\mathrm{H}^{2}(I)}^{2}
\end{aligned}
$$

Where in the last step we again made use of the Lemmata 1.6, 3.6 and 3.7.

In what follows we adapt some lemmata and theorems from [7] to get equivalent formulations for Assumption 3.1, as stated in Theorem 3.13.

Lemma 3.9. Let $q \in Q^{a d}, \delta q \in Q$ and $\left(\delta q_{n}\right)_{n \in \mathbb{N}} \subset Q$. If $\delta q_{n} \rightarrow \delta q$ in $\mathrm{C}^{1}(\bar{I})$ then it holds that

1. $S^{\prime}(q)\left(\delta q_{n}\right) \rightarrow S^{\prime}(q)(\delta q)$ in $\mathrm{H}^{1}\left(\Omega_{0}\right)$,

2. $S^{\prime \prime}(q)\left(\delta q_{n}, \delta q_{n}\right) \rightarrow S^{\prime \prime}(q)(\delta q, \delta q)$ in $\mathrm{H}^{1}\left(\Omega_{0}\right)$.

Proof. (1) For $\delta q_{n} \rightarrow \delta q$ in $\mathrm{C}^{1}(\bar{I})$ it immediately follows from (1.7) and (1.11) that the right hand side in (3.5) converges in $\mathrm{L}^{2}\left(\Omega_{0}\right)$. Hence, this part of the lemma follows with the standard $\mathrm{H}^{1}$-stability result.

(2) The second part is proven analogously to the first part. In order to show that the right hand side in (3.6) converges in $\mathrm{L}^{2}\left(\Omega_{0}\right)$, one has to make use of part (1) and the equations (1.7), (1.11) and (1.12).

Lemma 3.10. Let $q \in Q^{a d}, \delta q \in Q$ and $\left(\delta q_{n}\right)_{n \in \mathbb{N}} \subset Q$ and $\delta q_{n} \rightarrow \delta q$ in $\mathrm{C}^{1}(\bar{I})$. Let $m: Q^{a d} \times Q \mapsto \mathbb{R}$ and $n$ : $Q^{a d} \times Q \mapsto \mathbb{R}$ be defined by $m(q)(\delta q)=j^{\prime}(q)(\delta q)-\alpha\left(q^{\prime \prime}, \delta q^{\prime \prime}\right)_{\mathrm{L}^{2}(I)}$ and $n(q)(\delta q)=j^{\prime \prime}(q)(\delta q, \delta q)-\alpha\left(\delta q^{\prime \prime}, \delta q^{\prime \prime}\right)_{\mathrm{L}^{2}(I)}$, respectively. Then it holds that

1. $m(q)\left(\delta q_{n}\right) \rightarrow m(q)(\delta q)$ for $n \rightarrow \infty$,

2. $n(q)\left(\delta q_{n}\right) \rightarrow n(q)(\delta q)$ for $n \rightarrow \infty$.

Proof. This Lemma follows directly from the representations (3.8) and (3.9) in combination with Lemma 3.9 and (1.7).

Lemma 3.11. Let $q \in Q^{a d}, \delta q \in Q$ and $\left(\delta q_{n}\right)_{n \in \mathbb{N}} \subset Q$. If $\delta q_{n} \rightarrow \delta q$ in $\mathrm{H}^{2}(I)$, then

1. $j^{\prime}(q)\left(\delta q_{n}\right) \rightarrow j^{\prime}(q)(\delta q)$,

2. $j^{\prime \prime}(q)(\delta q, \delta q) \leq \liminf _{n \rightarrow \infty} j^{\prime \prime}(q)\left(\delta q_{n}, \delta q_{n}\right)$.

Proof. As $\mathrm{H}^{2}(I)$ is compactly embedded in $\mathrm{C}^{1}(\bar{I})$, we get $\delta q_{n} \rightarrow \delta q$ in $\mathrm{C}^{1}(\bar{I})$.

(1) This part follows from the fact that $\left(q^{\prime \prime}, \delta q_{n}^{\prime \prime}\right)_{\mathrm{L}^{2}(I)} \rightarrow\left(q^{\prime \prime}, \delta q^{\prime \prime}\right)_{\mathrm{L}^{2}(I)}$ and the first part of Lemma 3.10 .

(2) As the squared $\mathrm{H}^{2}(I)$-seminorm is a continuous and convex functional on $\mathrm{H}^{2}(I)$, it is also weakly lower semi-continuous, hence $\left(\delta q^{\prime \prime}, \delta q^{\prime \prime}\right)_{\mathrm{L}^{2}(I)} \leq \liminf _{n \rightarrow \infty}\left(\delta q_{n}^{\prime \prime}, \delta q_{n}^{\prime \prime}\right)_{\mathrm{L}^{2}(I)}$, and the second part follows with the second part of Lemma 3.10.

Lemma 3.12. Let $q \in Q^{a d}, \delta q_{n}, \delta q \in Q$ and $\delta q_{n} \rightarrow \delta q$ in $\mathrm{H}^{2}(I)$. If $\lim _{n \rightarrow \infty} j^{\prime \prime}(q)\left(\delta q_{n}, \delta q_{n}\right)=j^{\prime \prime}(q)(\delta q, \delta q)$, then $\delta q_{n} \rightarrow \delta q$ in $\mathrm{H}^{2}(I)$. 
Proof. At first we notice that $\delta q_{n} \rightarrow \delta q$ in $\mathrm{C}^{1}(\bar{I})$. With Lemma 3.10, (2), it follows that $\left\|\delta q_{n}^{\prime \prime}\right\|_{\mathrm{L}^{2}(I)} \rightarrow\left\|\delta q^{\prime \prime}\right\|_{\mathrm{L}^{2}(I)}$, hence we have convergence of the full $\mathrm{H}^{2}(I)$-norm. Strong convergence now follows from the convergence of the norm plus weak convergence.

Theorem 3.13. Let $\bar{q} \in Q^{\text {ad }}$ fulfill Assumption 3.1. Then there exists $\beta>0$ such that

$$
j^{\prime \prime}(\bar{q})(\delta q, \delta q) \geq \beta \cdot\|\delta q\|_{\mathrm{H}^{2}(I)}^{2} \quad \forall \delta q \in Q .
$$

Proof. Assume that (3.10) does not hold. Then there exists a sequence $\left(\delta q_{n}\right)_{n \in \mathbb{N}} \subset Q$ with $\left\|\delta q_{n}\right\|_{\mathrm{H}^{2}(I)}=1$ and

$$
j^{\prime \prime}(\bar{q})\left(\delta q_{n}, \delta q_{n}\right)<\frac{1}{n} .
$$

Possibly after extracting a subsequence we get the existence of an element $\overline{\delta q} \in Q$ with $\delta q_{n} \rightarrow \overline{\delta q}$ in $\mathrm{H}^{2}(I)$. We get

$$
0 \leq j^{\prime \prime}(\bar{q})(\overline{\delta q}, \overline{\delta q}) \leq \liminf _{n \rightarrow \infty} j^{\prime \prime}(\bar{q})\left(\delta q_{n}, \delta q_{n}\right) \leq \limsup _{n \rightarrow \infty} j^{\prime \prime}(\bar{q})\left(\delta q_{n}, \delta q_{n}\right) \leq \limsup _{n \rightarrow \infty} \frac{1}{n}=0,
$$

where the first inequality is just the necessary optimality condition of second order (3.2) and the second inequality is due to Lemma 3.11. Now, (3.11) leads to $j^{\prime \prime}(\bar{q})\left(\delta q_{n}, \delta q_{n}\right) \rightarrow j^{\prime \prime}(\bar{q})(\overline{\delta q}, \overline{\delta q})=0$. With Assumption 3.1 we get $\overline{\delta q}=0$, whereas Lemma 3.12 implies that $\delta q_{n} \rightarrow \overline{\delta q}$ in $\mathrm{H}^{2}(I)$. This is a contradiction to the fact that $\left\|\delta q_{n}\right\|_{\mathrm{H}^{2}(I)}=1$.

Due to the stability of $j^{\prime \prime}$ it is now possible to show coercivity also in a neighborhood of $\bar{q}$, as well as a quadratic growth condition.

Lemma 3.14. If $\bar{q}$ is a local solution of (1.3) fulfilling Assumption 3.1, then there exists $\delta_{1}>0$ such that, if $\|\bar{q}-p\|_{\mathrm{H}^{2}(I)} \leq \delta_{1}$ for $p \in Q^{a d}$, then it holds that

$$
j^{\prime \prime}(p)(\delta q, \delta q) \geq \frac{\beta}{2} \cdot\|\delta q\|_{\mathrm{H}^{2}(I)}^{2} \quad \forall \delta q \in Q .
$$

Proof. With Theorem 3.13 and Lemma 3.8 one gets

$$
\begin{aligned}
j^{\prime \prime}(p)(\delta q, \delta q) & =j^{\prime \prime}(\bar{q})(\delta q, \delta q)+j^{\prime \prime}(p)(\delta q, \delta q)-j^{\prime \prime}(\bar{q})(\delta q, \delta q) \\
& \geq j^{\prime \prime}(\bar{q})(\delta q, \delta q)-\left|j^{\prime \prime}(p)(\delta q, \delta q)-j^{\prime \prime}(\bar{q})(\delta q, \delta q)\right| \\
& \geq \beta \cdot\|\delta q\|_{\mathrm{H}^{2}(I)}^{2}-c \cdot\|\bar{q}-p\|_{\mathrm{H}^{2}(I)} \cdot\|\delta q\|_{\mathrm{H}^{2}(I)}^{2} \\
& =\left(\beta-c \cdot\|\bar{q}-p\|_{\mathrm{H}^{2}(I)}\right) \cdot\|\delta q\|_{\mathrm{H}^{2}(I)}^{2},
\end{aligned}
$$

and the result follows for $\delta_{1} \leq \frac{\beta}{2 c}$.

Lemma 3.15. Let $\bar{q}$ be a local minimum of (1.3) in which the Assumptions 1.5 and 3.1 hold. Then there exists $\delta_{2}>0$ such that for all $p \in Q^{a d}$ with $\|\bar{q}-p\|_{\mathrm{H}^{2}(I)} \leq \delta_{2}$ it holds that

$$
j(p) \geq j(\bar{q})+\frac{\beta}{2} \cdot\|\bar{q}-p\|_{\mathrm{H}^{2}(I)}^{2} .
$$

Proof. With Taylor we have for some $t \in[0,1]$ :

$$
\begin{aligned}
j(p) & =j(\bar{q})+j^{\prime}(\bar{q})(p-\bar{q})+j^{\prime \prime}(\bar{q}+t \cdot(p-\bar{q}))(p-\bar{q}, p-\bar{q}) \\
& =j(\bar{q})+j^{\prime \prime}(\bar{q}+t \cdot(p-\bar{q}))(p-\bar{q}, p-\bar{q}) \\
& \geq j(\bar{q})+\frac{\beta}{2} \cdot\|\bar{q}-p\|_{\mathrm{H}^{2}(I)}^{2},
\end{aligned}
$$

whereas in the second step we used the first order optimality condition (3.1) in $\bar{q}$, in the third step we used Lemma 3.14 .

Before a priori error estimates can be established, we need some higher regularity of the optimal control $\bar{q}$. 


\subsection{Higher regularity of the optimal solution}

In this subsection we investigate the regularity of the optimal solutions $(\bar{q}, \bar{u}) \in \mathrm{H}^{2}(I) \times \mathrm{H}^{3 / 2}\left(\Omega_{\bar{q}}\right)$ of $(1.3)$. The main result of this subsection will be the following.

Theorem 3.16. Every solution $(\bar{q}, \bar{u})$ of (1.3) in which Assumption 1.5 holds possesses the higher regularity $(\bar{q}, \bar{u}) \in \mathrm{H}^{4}(I) \times \mathrm{H}^{2}\left(\Omega_{\bar{q}}\right)$.

In order to prove this theorem, we first have a look at the regularity of the transported solution $S(q)$ and its derivatives.

Lemma 3.17. For $q \in Q^{\text {ad }}$ it holds that $S(q)$ is uniformly bounded in $\mathrm{H}^{5 / 4}\left(\Omega_{0}\right)$.

Proof. As $\mathrm{H}^{2}(I) \hookrightarrow \mathrm{C}^{1,1 / 2}(\bar{I})$, the coefficients of the matrix $A_{q}$ are elements of $\mathrm{C}^{0,1 / 2}\left(\overline{\Omega_{0}}\right)$. Hence we can apply Theorem 1.12, (1), which leads to $S(q) \in \mathrm{H}^{5 / 4}\left(\Omega_{0}\right)$. The uniform boundedness follows from the boundedness of the right hand side in $(3.4)$ in $\mathrm{L}^{2}\left(\Omega_{0}\right)$, which is due to Lemma 1.6.

Lemma 3.18. For $q \in Q^{a d}, \delta q \in Q$ it holds with $q$-independent constants

$$
\left\|S^{\prime}(q)(\delta q)\right\|_{\mathrm{W}_{0}^{1,8 / 7}\left(\Omega_{0}\right)} \leq c \cdot\|\delta q\|_{\mathrm{H}^{1}(I)} .
$$

Proof. For $q \in Q^{a d}$ and $\delta q \in Q$ let $g(q, \delta q)(\cdot) \in \mathrm{W}^{-1,8 / 7}\left(\Omega_{0}\right)$ be defined via

$$
g(q, \delta q)(z)=\left(\left(\nabla f \circ T_{q}\right)^{T} \cdot V_{\delta q}, z \gamma_{q}\right)+\left(f \circ T_{q}, z \operatorname{div}\left(V_{\delta q}\right)\right)-\left(\nabla S(q), A_{q, \delta q}^{\prime} \nabla z\right)-\left(S(q), z \operatorname{div}\left(V_{\delta q}\right)\right) .
$$

Now we use the Lemmata 1.6, 1.7 as well as the definitions (1.7) and (1.11) to estimate the norm

$$
\begin{aligned}
& \|g(q, \delta q)(\cdot)\|_{\mathrm{W}^{-1,8 / 7}\left(\Omega_{0}\right)}=\sup _{\|z\|_{\mathrm{W}_{0}^{1,8}\left(\Omega_{0}\right)}=1} g(q, \delta q)(z) \\
& =\sup _{\|z\|_{\mathrm{W}_{0}^{1,8}\left(\Omega_{0}\right)}=1}\left(\left(\nabla f \circ T_{q}\right)^{T} \cdot V_{\delta q}, z \gamma_{q}\right)+\left(f \circ T_{q}, z \operatorname{div}\left(V_{\delta q}\right)\right)-\left(\nabla S(q), A_{q, \delta q}^{\prime} \nabla z\right)-\left(S(q), z \operatorname{div}\left(V_{\delta q}\right)\right) \\
& \leq \sup _{\|z\|_{\mathrm{W}_{0}^{1,8}\left(\Omega_{0}\right)}=1} c \cdot\|\delta q\|_{\mathrm{H}_{0}^{1}(I)}+\|\nabla S(q)\|_{\mathrm{L}^{8 / 3}\left(\Omega_{0}\right)} \cdot\left\|A_{q, \delta q}^{\prime}\right\|_{\mathrm{L}^{2}\left(\Omega_{0}\right)} \cdot\|\nabla z\|_{\mathrm{L}^{8}\left(\Omega_{0}\right)}+\|S(q)\|_{\mathrm{L}^{2}\left(\Omega_{0}\right)} \cdot\|z\|_{\mathrm{L}^{2}\left(\Omega_{0}\right)} \cdot\|\delta q\|_{\mathrm{H}^{1}(I)} \\
& \leq \sup _{\|z\|_{\mathrm{W}_{0}^{1,8}\left(\Omega_{0}\right)}=1} c \cdot\|\delta q\|_{\mathrm{H}^{1}(I)} \cdot\left(1+\|z\|_{\mathrm{W}_{0}^{1,8}\left(\Omega_{0}\right)}\right) \\
& =c \cdot\|\delta q\|_{\mathrm{H}^{1}(I)}
\end{aligned}
$$

where in the last steps we used Lemma 3.17 in combination with the continuous embedding $\mathrm{H}^{5 / 4}\left(\Omega_{0}\right) \hookrightarrow$ $\mathrm{W}^{1,8 / 3}\left(\Omega_{0}\right)$ as well as the generalized Hölder's inequality.

Because of $\mathrm{W}_{0}^{1,8}\left(\Omega_{0}\right) \hookrightarrow \mathrm{H}_{0}^{1}\left(\Omega_{0}\right)$ and (3.5) it follows that $S^{\prime}(q)(\delta q)$ uniquely solves

$$
a(q)\left(S^{\prime}(q)(\delta q), z\right)=g(q, \delta q)(z) \quad \forall z \in \mathrm{W}_{0}^{1,8}\left(\Omega_{0}\right) .
$$

With [3], Theorem 1, it follows that

$$
\left\|S^{\prime}(q)(\delta q)\right\|_{\mathrm{W}_{0}^{1,8 / 7}\left(\Omega_{0}\right)} \leq c \cdot\|g(q, \delta q)(\cdot)\|_{\mathrm{W}^{-1,8 / 7}\left(\Omega_{0}\right)} \leq c \cdot\|\delta q\|_{\mathrm{H}^{1}(I) .}
$$


Lemma 3.19. The optimal solution $\bar{q}$ has the higher regularity $\bar{q} \in \mathrm{H}^{3}(I) \hookrightarrow \mathrm{C}^{2,1 / 2}(\bar{I})$.

Proof. In (3.8) we have already shown that

$$
\begin{aligned}
j^{\prime}(q)(\delta q)= & \underbrace{\frac{1}{2}\left(S(q)-u_{d} \circ T_{q},\left(S(q)-u_{d} \circ T_{q}\right) \operatorname{div}\left(V_{\delta q}\right)\right)+\left(S^{\prime}(q)(\delta q)-\left(\nabla u_{d} \circ T_{q}\right)^{T} \cdot V_{\delta q},\left(S(q)-u_{d} \circ T_{q}\right) \gamma_{q}\right)}_{=j_{1}(\delta q)} \\
& +\alpha\left(q^{\prime \prime}, \delta q^{\prime \prime}\right)_{\mathrm{L}^{2}(I)} .
\end{aligned}
$$

The functional $j_{1}$ as defined in (3.12) is linear in $\delta q$. Then, by using (1.7), Lemmas 1.6 and 3.17 with the embedding $\mathrm{H}^{5 / 4}\left(\Omega_{0}\right) \hookrightarrow \mathrm{L}^{\infty}\left(\Omega_{0}\right)$ as well as Lemma 3.18 we conclude that

$$
j_{1}(\delta q) \leq c \cdot\|\delta q\|_{\mathrm{H}_{0}^{1}(I)},
$$

where $c=c\left(q, u_{d}, f\right)$. The Riesz Representation Theorem now yields the existence of $r \in \mathrm{H}_{0}^{1}(I)$ such that

$$
j^{\prime}(q)(\delta q)=\int_{0}^{1} r^{\prime} \delta q^{\prime} \mathrm{d} x+\alpha \int_{0}^{1} q^{\prime \prime} \delta q^{\prime \prime} \mathrm{d} x \quad \forall \delta q \in Q .
$$

Now we recall the first order optimality conditions in $\bar{q}$,

$$
j^{\prime}(\bar{q})(\delta q)=0 \quad \forall \delta q \in Q
$$

and because of $\mathrm{C}_{0}^{\infty}(\bar{I}) \subset Q$ we a fortiori get

$$
j^{\prime}(\bar{q})(\delta q)=0 \quad \forall \delta q \in \mathrm{C}_{0}^{\infty}(\bar{I})
$$

and therefore

$$
\int_{0}^{1}\left(\alpha \bar{q}^{\prime \prime}-r\right) \delta q^{\prime \prime} \mathrm{d} x=0 \quad \forall \delta q \in \mathrm{C}_{0}^{\infty}(\bar{I}) .
$$

Hence, $\alpha \bar{q}^{\prime \prime}-r=0$ in $\mathrm{H}_{0}^{2}(I) \subset \mathrm{H}_{0}^{1}(I)$. Because of $r \in \mathrm{H}_{0}^{1}(I)$ we get $\alpha \bar{q}^{\prime \prime} \in \mathrm{H}_{0}^{1}(I)$ and hence $\bar{q} \in \mathrm{H}^{3}(I)$.

With the previous Lemma it now follows that the coefficients of the matrix $A_{\bar{q}}$ are Lipschitz continuous. The following corollary thus follows with Theorem 1.12, (3).

Corollary 3.20. It holds that $\bar{u}^{\bar{q}}=S(\bar{q}) \in \mathrm{H}^{2}\left(\Omega_{0}\right), \bar{z}^{\bar{q}} \in \mathrm{H}^{2}\left(\Omega_{0}\right)$, where $\bar{z}^{\bar{q}}$ is the transported adjoint state as defined in (1.6), associated to $\bar{u}^{\bar{q}}$.

In order to prove that the "original" state $\bar{u}$ also possesses this higher regularity we need the following lemma.

Lemma 3.21. If $v \circ T_{\bar{q}}=v^{\bar{q}} \in \mathrm{H}^{2}\left(\Omega_{0}\right)$, then it holds that $v \in \mathrm{H}^{2}\left(\Omega_{\bar{q}}\right)$.

Proof. Because of $\bar{q} \in \mathrm{H}^{3}(I) \hookrightarrow \mathrm{C}^{2,1 / 2}(\bar{I})$ we also have

$$
T_{\bar{q}}^{-1}=\left(\begin{array}{c}
x \\
y-(1-y) \frac{\bar{q}(x)}{1-\bar{q}(x)}
\end{array}\right)=\left(\begin{array}{l}
T_{1} \\
T_{2}
\end{array}\right) \in\left(\mathrm{C}^{2}\left(\bar{\Omega}_{\bar{q}}\right)\right)^{2} .
$$


A formal calculation yields

$$
\begin{aligned}
v & =v^{\bar{q}} \circ T_{\bar{q}}^{-1}, \\
\nabla v & =\left(\mathrm{D}\left(T_{\bar{q}}^{-1}\right)\right)^{T} \cdot \nabla v^{\bar{q}} \circ T_{\bar{q}}^{-1}, \\
\nabla^{2} v & =\left(\frac{\partial}{\partial x} v^{\bar{q}}\right) \circ T_{\bar{q}}^{-1} \cdot \nabla^{2} T_{1}+\left(\frac{\partial}{\partial y} v^{\bar{q}}\right) \circ T_{\bar{q}}^{-1} \cdot \nabla^{2} T_{2}+\left(\mathrm{D}\left(T_{\bar{q}}^{-1}\right)\right)^{T} \cdot \nabla^{2} v^{\bar{q}} \circ T_{\bar{q}}^{-1} \cdot \mathrm{D}\left(T_{\bar{q}}^{-1}\right) .
\end{aligned}
$$

From $\mathrm{H}^{2}\left(\Omega_{0}\right) \hookrightarrow \mathrm{C}^{0,1-\varepsilon}\left(\bar{\Omega}_{0}\right),(3.13)$ and (3.14) it follows that $v \in \mathrm{L}^{2}\left(\Omega_{\bar{q}}\right)$. Furthermore, with (3.15) it holds that

$$
\begin{aligned}
\int_{\Omega_{\bar{q}}}|\nabla v|^{2} \mathrm{~d} x & =\int_{\Omega_{\bar{q}}}\left|\mathrm{D}\left(T_{\bar{q}}^{-1}\right)^{T} \cdot \nabla v^{\bar{q}} \circ T_{\bar{q}}^{-1}\right|^{2} \mathrm{~d} x \\
& =\int_{\Omega_{0}}\left|\mathrm{D}\left(T_{\bar{q}}\right)^{-1} \cdot \nabla v^{\bar{q}}\right|^{2} \gamma_{\bar{q}} \mathrm{~d} x \leq c \cdot \int_{\Omega_{0}}\left|\nabla v^{\bar{q}}\right|^{2} \mathrm{~d} x<\infty,
\end{aligned}
$$

and hence $\nabla v \in \mathrm{L}^{2}\left(\Omega_{\bar{q}}\right)$. It remains to bound the second derivatives.

$$
\int_{\Omega_{\bar{q}}}\left|\left(\frac{\partial}{\partial x} v^{\bar{q}}\right) \circ T_{\bar{q}}^{-1} \cdot \nabla^{2} T_{1}\right|^{2} \mathrm{~d} x=\int_{\Omega_{0}}\left|\left(\frac{\partial}{\partial x} v^{\bar{q}}\right) \cdot \nabla^{2} T_{1} \circ T_{\bar{q}}\right|^{2} \gamma_{\bar{q}} \mathrm{~d} x \leq c \cdot \int_{\Omega_{0}}\left(\frac{\partial}{\partial x} v^{\bar{q}}\right)^{2} \mathrm{~d} x<\infty,
$$

where we used the fact that $\nabla^{2} T_{1}$ and $T_{\bar{q}}$ are continuous and $\frac{\partial}{\partial x} v^{\bar{q}} \in \mathrm{L}^{2}\left(\Omega_{0}\right)$. The proof that the second part of the sum in (3.16) is finite is done analogously. Finally we have

$$
\begin{aligned}
\int_{\Omega_{\bar{q}}}\left|\left(\mathrm{D}\left(T_{\bar{q}}^{-1}\right)\right)^{T} \cdot \nabla^{2} v^{\bar{q}} \circ T_{\bar{q}}^{-1} \cdot \mathrm{D}\left(T_{\bar{q}}^{-1}\right)\right|^{2} \mathrm{~d} x & =\int_{\Omega_{0}}\left|\left(\mathrm{D}\left(T_{\bar{q}}^{-1}\right)\right)^{T} \circ T_{\bar{q}} \cdot \nabla^{2} v^{\bar{q}} \cdot \mathrm{D}\left(T_{\bar{q}}^{-1}\right) \circ T_{\bar{q}}\right|^{2} \gamma_{\bar{q}} \mathrm{~d} x \\
& \leq c \cdot \int_{\Omega_{0}}\left|\nabla^{2} v^{\bar{q}}\right|^{2} \mathrm{~d} x<\infty,
\end{aligned}
$$

where we again used $(3.13)$ and $\nabla^{2} v^{\bar{q}} \in \mathrm{L}^{2}\left(\Omega_{0}\right)$.

Corollary 3.22. It holds that $\bar{u}, \bar{z} \in \mathrm{H}^{2}\left(\Omega_{\bar{q}}\right)$.

With this improved regularity of $\bar{q}, \bar{u}$ and $\bar{z}$ it now follows that the moving part of the boundary $\Gamma_{\bar{q}}$ is $\mathrm{C}^{2}$, hence the boundary integral representation (1.5) actually holds in $\bar{q}$. Next we have a closer look at that representation of $j^{\prime}$. Again, we exploit the first order optimality conditions restricted to the $\operatorname{set}_{0}^{\infty}(\bar{I}) \subset Q$.

$$
j^{\prime}(\bar{q})(\delta q)=0 \quad \forall \delta q \in \mathrm{C}_{0}^{\infty}(I) .
$$

Now we analyze (3.17). First of all, we have

$$
n(x)=\frac{1}{\sqrt{\bar{q}^{\prime}(x)^{2}+1}}\left(\begin{array}{c}
\bar{q}^{\prime}(x) \\
-1
\end{array}\right)
$$

for the normalized outer normal on that part of the boundary $\Gamma_{\bar{q}}$ which is described by $\bar{q}$. If $x \in\{0,1\}$ or $y=1$ we have $V_{\bar{q}, \delta q}=(0,0)^{T}$, so the derivative (1.5) just consists of an integral over the "moving" part $\bar{q}$ of the boundary. This part of $\Gamma_{\bar{q}}$ is parametrized by the curve

$$
\begin{array}{rlrl}
\gamma:[0,1] & \mapsto \mathbb{R}^{2}, & t & \mapsto\left(\begin{array}{c}
t \\
\bar{q}(t)
\end{array}\right), \\
\gamma^{\prime}(t) & =\left(\begin{array}{c}
1 \\
\bar{q}^{\prime}(t)
\end{array}\right), & \left\|\gamma^{\prime}(t)\right\|=\sqrt{\bar{q}^{\prime}(t)^{2}+1}
\end{array}
$$


Therefore

$$
\begin{aligned}
j^{\prime}(\bar{q})(\delta q)= & \int_{\Gamma_{\bar{q}}}\left(\frac{1}{2}\left(\bar{u}-u_{d}\right)^{2}+\partial_{n} \bar{z} \partial_{n} \bar{u}\right) \frac{1}{\sqrt{\bar{q}^{\prime 2}+1}}(\bar{q}-1) \frac{\delta q}{1-\bar{q}} \mathrm{~d} \Gamma_{\bar{q}}(x)+\alpha \int_{0}^{1} \bar{q}^{\prime \prime} \delta q^{\prime \prime} \mathrm{d} x \\
= & -\int_{0}^{1} \underbrace{\frac{1}{2}\left(\bar{u}(x, \bar{q}(x))-u_{d}(x, \bar{q}(x))\right)^{2}}_{=h_{1}(x)} \delta q(x) \mathrm{d} x \\
& -\int_{0}^{1} \underbrace{\frac{1}{\bar{q}^{\prime}(x)^{2}+1}\left(\left(\begin{array}{c}
\bar{q}^{\prime}(x) \\
-1
\end{array}\right) \cdot \nabla \bar{z}(x, \bar{q}(x))\right) \cdot\left(\left(\begin{array}{c}
\bar{q}^{\prime}(x) \\
-1
\end{array}\right) \cdot \nabla \bar{u}(x, \bar{q}(x))\right)}_{=h_{2}(x)} \delta q(x) \mathrm{d} x+\alpha \int_{0}^{1} \bar{q}^{\prime \prime} \delta q^{\prime \prime} \mathrm{d} x .
\end{aligned}
$$

As $\bar{u}, \bar{z} \in \mathrm{H}^{2}\left(\Omega_{q}\right)$ we have $\bar{u}, \nabla \bar{u}, \nabla \bar{z} \in \mathrm{H}^{1 / 2}\left(\Gamma_{q}\right) \hookrightarrow \mathrm{L}^{4}\left(\Gamma_{\bar{q}}\right)$ as follows from the Trace Theorem [27] and hence $h_{1}, h_{2} \in \mathrm{L}^{2}(I)$. By setting $h=h_{1}+h_{2}$ we now get that (3.17) is equivalent to

$$
\int_{0}^{1} \bar{q}^{\prime \prime} \delta q^{\prime \prime} \mathrm{d} x=\int_{0}^{1} \frac{1}{\alpha} h \delta q \mathrm{~d} x \quad \forall \delta q \in \mathrm{C}_{0}^{\infty}(I) .
$$

Equation (3.18) is just the definition of the fourth weak derivative of $\bar{q}$, and from $\frac{1}{\alpha} h \in \mathrm{L}^{2}(I)$ we deduce $\bar{q} \in \mathrm{H}^{4}(I)$. This completes the proof of Theorem 3.16.

Remark 3.23. Due to this improved regularity of $\bar{q}$ it is possible to use partial integration within (3.18) with test functions $\delta q \in Q$ to show that $\bar{q}$ is a solution of the following fourth order boundary value problem.

$$
\begin{aligned}
\bar{q}^{(i v)} & =\frac{1}{\alpha} h, \quad \text { in }(0,1), \\
\bar{q}(0) & =\bar{q}(1)=0, \\
\bar{q}^{\prime \prime}(0) & =\bar{q}^{\prime \prime}(1)=0 .
\end{aligned}
$$

Remark 3.24. The embedding $\mathrm{H}^{1 / 2}\left(\Gamma_{\bar{q}}\right) \hookrightarrow \mathrm{L}^{p}\left(\Gamma_{\bar{q}}\right), \forall p<\infty$, implies that $h \in \mathrm{L}^{p}(I)$ for arbitrary $p<\infty$, which leads to the even higher regularity $\bar{q} \in \mathrm{W}^{4, p}(I) \hookrightarrow \mathrm{C}^{3,1-\varepsilon}(\bar{I})$ for $\varepsilon>0$.

Remark 3.25. The proof of Theorem 3.16 just relied on an exploitation of the first order optimality condition (3.1), therefore it holds not only for global solutions of (1.3) but also for local ones which fulfill Assumption 1.5 .

\subsection{Estimation of the error due to the discretization of the control}

In order to proof the existence of a sequence $(\bar{q})_{\sigma>0}$ as predicted in (3.3), one first needs to show that there exist functions from the discretized control space near $\bar{q}$. We therefore construct an interpolation operator $i_{\sigma}$ for the optimal solution $\bar{q}$.

Definition 3.26. Let $i_{\sigma}: Q \mapsto Q_{\sigma}, f \mapsto i_{\sigma} f$ be a Hermite interpolation operator with the following properties.

- $\left(i_{\sigma} f\right)\left(x_{i}\right)=f\left(x_{i}\right),\left(i_{\sigma} f\right)^{\prime}\left(x_{i}\right)=f^{\prime}\left(x_{i}\right), \quad \forall i \in\{0, \ldots, N\}$,

- $\left.i_{\sigma} f\right|_{I_{i}} \in \mathcal{P}_{3}\left(I_{i}\right) \quad \forall i \in\{1, \ldots, N\}$.

As every polynomial of degree 3 over an interval is uniquely defined by its functional values and the values of its derivative at the endpoints of that interval, the operator $i_{\sigma}$ is well-defined for each $f \in \mathrm{C}^{1}(\bar{I})$. With the help of the Bramble-Hilbert Lemma [5] and Theorem 3.16 it can be shown that

Corollary 3.27. The interpolation error for the optimal control $\bar{q}$ can be estimated by

$$
\left\|\bar{q}-i_{\sigma} \bar{q}\right\|_{\mathrm{H}^{2}(I)} \leq c \cdot \sigma^{2} \cdot|\bar{q}|_{\mathrm{H}^{4}(I)},
$$

where the constant $c$ is independent of $\sigma$ and $\bar{q}$. 
As already mentioned, it is necessary to show that for $\sigma$ sufficiently small, there exists a sequence $\left(\bar{q}_{\sigma}\right)_{\sigma>0}$ of local optimal solutions of (2.3) with

$$
\left\|\bar{q}-\bar{q}_{\sigma}\right\|_{\mathrm{H}^{2}(I)} \rightarrow 0 \quad \text { for } \sigma \rightarrow 0 .
$$

We call $\bar{q}_{\sigma}$ local optimal for $(2.3)$ if there exists $\varepsilon>0$ with

$$
j\left(\bar{q}_{\sigma}\right) \leq j\left(q_{\sigma}\right) \quad \forall q_{\sigma} \in Q_{\sigma}^{a d},\left\|q_{\sigma}-\bar{q}_{\sigma}\right\|_{\mathrm{H}^{2}(I)} \leq \varepsilon .
$$

In what follows we adapt the steps undertaken in [6]. First of all, for a given $\varepsilon>0$, let

$$
Q_{\sigma, \varepsilon}^{a d}=\left\{q_{\sigma} \in Q_{\sigma}^{a d} \mid\left\|q_{\sigma}-\bar{q}\right\|_{\mathrm{H}^{2}(I)} \leq \varepsilon\right\},
$$

and introduce an auxiliary problem as follows.

$$
\text { Minimize } j\left(q_{\sigma, \varepsilon}\right), q_{\sigma, \varepsilon} \in Q_{\sigma, \varepsilon}^{a d} \text {. }
$$

Let $\varepsilon>0$ be fixed such that Lemma 3.15 is applicable for all $p \in Q_{\sigma, \varepsilon}^{a d}$, and choose $\sigma>0$ sufficiently small such that $i_{\sigma} \bar{q} \in Q_{\sigma, \varepsilon}^{a d} \neq \emptyset$. Similar to Theorem 2.3 one can show that (3.21) has at least one global solution $\bar{q}_{\sigma, \varepsilon}$.

The next steps are undertaken in order to show that $\bar{q}_{\sigma, \varepsilon}$ is also a local solution of (2.3).

Lemma 3.28. For $\sigma \rightarrow 0$ it holds that $j\left(\bar{q}_{\sigma, \varepsilon}\right) \rightarrow j(\bar{q})$.

Proof. We have

$$
j\left(\bar{q}_{\sigma, \varepsilon}\right) \geq j(\bar{q}),
$$

by the definition of $\bar{q}$. But due to the definition of $\bar{q}_{\sigma, \varepsilon}$ we also have

$$
j\left(\bar{q}_{\sigma, \varepsilon}\right)-j(\bar{q}) \leq j\left(i_{\sigma} \bar{q}\right)-j(\bar{q}) \leq c \cdot\left\|i_{\sigma} \bar{q}-\bar{q}\right\|_{\mathrm{H}^{2}(I)} \rightarrow 0 \quad \text { for } \sigma \rightarrow 0,
$$

where we also used the Lemmata 3.8 and Corollary 3.27 .

Lemma 3.29. For $\sigma \rightarrow 0$ it holds that $\left\|\bar{q}_{\sigma, \varepsilon}-\bar{q}\right\|_{\mathrm{H}^{2}(I)} \rightarrow 0$.

Proof. With Lemma 3.15 we get

$$
\underbrace{j\left(\bar{q}_{\sigma, \varepsilon}\right)-j(\bar{q})}_{\rightarrow 0} \geq \frac{\beta}{2} \cdot\left\|\bar{q}_{\sigma, \varepsilon}-\bar{q}\right\|_{\mathrm{H}^{2}(I)}^{2} .
$$

Lemma 3.30. For $\sigma$ sufficiently small, $\bar{q}_{\sigma, \varepsilon}$ is a local solution of (2.3).

Proof. We have to show that for $\sigma$ small enough all $q_{\sigma} \in Q_{\sigma}^{a d}$ which are sufficiently close to $\bar{q}_{\sigma, \varepsilon}$ are also elements of $Q_{\sigma, \varepsilon}^{a d}$. Hence choose $\sigma$ such that $\left\|\bar{q}_{\sigma, \varepsilon}-\bar{q}\right\|_{\mathrm{H}^{2}(I)} \leq \frac{\varepsilon}{2}$. Now it holds for all $q_{\sigma} \in Q_{\sigma}^{a d}$ with $\left\|\bar{q}_{\sigma, \varepsilon}-q_{\sigma}\right\|_{\mathrm{H}^{2}(I)} \leq \frac{\varepsilon}{2}$ that

$$
\left\|\bar{q}-q_{\sigma}\right\|_{\mathrm{H}^{2}(I)} \leq\left\|\bar{q}-\bar{q}_{\sigma, \varepsilon}\right\|_{\mathrm{H}^{2}(I)}+\left\|\bar{q}_{\sigma, \varepsilon}-q_{\sigma}\right\|_{\mathrm{H}^{2}(I)} \leq \varepsilon
$$

and this yields

$$
q_{\sigma} \in Q_{\sigma, \varepsilon}^{a d}
$$


Corollary 3.31. Let $\bar{q}_{\sigma}$ be a solution of (3.21). Then, for all sufficiently small $\sigma$ it holds that

$$
j^{\prime}\left(\bar{q}_{\sigma}\right)\left(\delta q_{\sigma}\right)=0 \quad \forall \delta q_{\sigma} \in Q_{\sigma} .
$$

Proof. Due to Lemma 3.29, Assumption 1.5 holds for $\bar{q}_{\sigma}$ and (3.22) follows with Lemma 3.30.

Now we can proof the first part of Theorem 3.2. We have

$$
\left\|\bar{q}-\bar{q}_{\sigma}\right\|_{\mathrm{H}^{2}(I)} \leq\left\|\bar{q}-i_{\sigma} \bar{q}\right\|_{\mathrm{H}^{2}(I)}+\left\|i_{\sigma} \bar{q}-\bar{q}_{\sigma}\right\|_{\mathrm{H}^{2}(I)} .
$$

With Corollary 3.27 it follows that

$$
\left\|\bar{q}-i_{\sigma} \bar{q}\right\|_{\mathrm{H}^{2}(I)} \leq c \cdot \sigma^{2},
$$

hence it remains to estimate the latter part on the right hand side in (3.23). With the Mean Value Theorem and Lemma 3.14 we have for some $t \in[0,1]$ and $\xi=t \cdot i_{\sigma} \bar{q}+(1-t) \cdot \bar{q}_{\sigma}$ :

$$
\begin{aligned}
\frac{\beta}{2} \cdot\left\|i_{\sigma} \bar{q}-\bar{q}_{\sigma}\right\|_{\mathrm{H}^{2}(I)}^{2} & \leq j^{\prime \prime}(\xi)\left(i_{\sigma} \bar{q}-\bar{q}_{\sigma}, i_{\sigma} \bar{q}-\bar{q}_{\sigma}\right) \\
& =j^{\prime}\left(i_{\sigma} \bar{q}\right)\left(i_{\sigma} \bar{q}-\bar{q}_{\sigma}\right)-j^{\prime}\left(\bar{q}_{\sigma}\right)\left(i_{\sigma} \bar{q}-\bar{q}_{\sigma}\right) \\
& =j^{\prime}\left(i_{\sigma} \bar{q}\right)\left(i_{\sigma} \bar{q}-\bar{q}_{\sigma}\right)-j^{\prime}(\bar{q})\left(i_{\sigma} \bar{q}-\bar{q}_{\sigma}\right),
\end{aligned}
$$

where we used the fact that $j^{\prime}(\bar{q})\left(i_{\sigma} \bar{q}-\bar{q}_{\sigma}\right)=j^{\prime}\left(\bar{q}_{\sigma}\right)\left(i_{\sigma} \bar{q}-\bar{q}_{\sigma}\right)=0$, due to Assumption 1.5 and Corollary 3.31 . With Corollary 3.27 and Lemma 3.8 we continue with

$$
\begin{aligned}
j^{\prime}\left(i_{\sigma} \bar{q}\right)\left(i_{\sigma} \bar{q}-\bar{q}_{\sigma}\right)-j^{\prime}(\bar{q})\left(i_{\sigma} \bar{q}-\bar{q}_{\sigma}\right) & \leq c \cdot\left\|\bar{q}-i_{\sigma} \bar{q}\right\|_{\mathrm{H}^{2}(I)} \cdot\left\|i_{\sigma} \bar{q}-\bar{q}_{\sigma}\right\|_{\mathrm{H}^{2}(I)} \\
& \leq c \cdot \sigma^{2} \cdot\left\|i_{\sigma} \bar{q}-\bar{q}_{\sigma}\right\|_{\mathrm{H}^{2}(I)},
\end{aligned}
$$

and so we arrive at

$$
\left\|i_{\sigma} \bar{q}-\bar{q}_{\sigma}\right\|_{\mathrm{H}^{2}(I)} \leq c \cdot \sigma^{2} .
$$

\subsection{Estimation of the error due to the discretization of the state}

In this subsection, some effort is put into deriving error estimates between the operator $S$ and its discrete analogue $S_{h}$. First of all recall the definition of the operator $S_{h}$ and its derivatives, the existence follows analogously to $S$ (cf. Cor. 3.4).

1. $u_{h}=S_{h}(q) \in V_{h}$ is the solution of

$$
a(q)\left(u_{h}, v_{h}\right)=\left(f \circ T_{q}, v_{h} \gamma_{q}\right) \quad \forall v_{h} \in V_{h} .
$$

2. $\delta u_{h}=S_{h}^{\prime}(q)(\delta q) \in V_{h}$ is the solution of

$$
\begin{aligned}
a(q)\left(\delta u_{h}, v_{h}\right)= & \left(\left(\nabla f \circ T_{q}\right)^{T} \cdot V_{\delta q}, v_{h} \gamma_{q}\right)+\left(f \circ T_{q}, v_{h} \operatorname{div}\left(V_{\delta q}\right)\right) \\
& -\left(\nabla u_{h}, A_{q, \delta q}^{\prime} \nabla v_{h}\right)-\left(u_{h}, v_{h} \operatorname{div}\left(V_{\delta q}\right)\right) \quad \forall v_{h} \in V_{h} .
\end{aligned}
$$

3. $\tau u_{h}=S_{h}^{\prime \prime}(q)(\delta q, \delta q) \in V_{h}$ is the solution of

$$
\begin{aligned}
a(q)\left(\tau u_{h}, v_{h}\right)= & \left(V_{\delta q}^{T} \cdot \nabla^{2} f \circ T_{q} \cdot V_{\delta q}, v_{h} \gamma_{q}\right)+2\left(\left(\nabla f \circ T_{q}\right)^{T} \cdot V_{\delta q}, v_{h} \operatorname{div}\left(V_{\delta q}\right)\right) \\
& -\left(\nabla u_{h}, A_{q, \delta q, \delta q}^{\prime \prime} \nabla v_{h}\right)-2\left(\nabla \delta u_{h}, A_{q, \delta q}^{\prime} \nabla v_{h}\right)-\left(\delta u_{h}, v_{h} \operatorname{div}\left(V_{\delta q}\right)\right) \quad \forall v_{h} \in V_{h} .
\end{aligned}
$$

Remark 3.32. We would like to point out that all constants in the following estimates are of course independent of $\sigma$. This follows mainly from Theorem 1.12, part (3), Lemma 1.9 as well as Corollary 3.38. 
Lemma 3.33. For $q \in Q^{a d}, \delta q \in Q$ it holds that

1. $\left\|S_{h}(q)\right\|_{\mathrm{H}^{1}\left(\Omega_{0}\right)} \leq c$,

2. $\left\|S_{h}^{\prime}(q)(\delta q)\right\|_{\mathrm{H}^{1}\left(\Omega_{0}\right)} \leq c \cdot\|\delta q\|_{\mathrm{H}^{2}(I)}$.

Proof. To proof this lemma one has to proceed similar to the proof of Lemma 3.6.

In what follows we will prove some error estimates for the error between the continuous state and its derivatives and their discrete counterparts.

The following corollary, which follows by a direct computation, will be needed within the proof of the Lemmata 3.35 and 3.39.

Corollary 3.34. For $A \in \mathbb{R}^{n \times n}$ and $b \in \mathbb{R}^{n}$ it holds that

$$
\operatorname{div}(A \cdot b)=A^{T} \bullet \nabla b+\left(\operatorname{div}\left(A^{T}\right)\right)^{T} \cdot b,
$$

where for $X, Y \in \mathbb{R}^{n \times n}$ we set

$$
X \bullet Y=\sum_{i, j=1}^{n} x_{i, j} y_{i, j}=\operatorname{trace}\left(X^{T} \cdot Y\right),
$$

and the divergence of a matrix $X$ is taken for each row separately.

Lemma 3.35. For $q_{\sigma} \in Q_{\sigma}^{a d}, \delta q \in Q$ and $k \in\{0,1\}$ it holds that

1. $\left\|S\left(q_{\sigma}\right)-S_{h}\left(q_{\sigma}\right)\right\|_{\mathrm{H}^{1-k}\left(\Omega_{0}\right)} \leq c \cdot h^{1+k}$,

2. $\left\|S^{\prime}\left(q_{\sigma}\right)(\delta q)-S_{h}^{\prime}\left(q_{\sigma}\right)(\delta q)\right\|_{\mathrm{H}^{1}\left(\Omega_{0}\right)} \leq c \cdot h^{1 / 4} \cdot\|\delta q\|_{\mathrm{H}^{2}(I)}$,

3. $\left\|S^{\prime \prime}\left(q_{\sigma}\right)(\delta q, \delta q)-S_{h}^{\prime \prime}\left(q_{\sigma}\right)(\delta q, \delta q)\right\|_{\mathrm{L}^{2}\left(\Omega_{0}\right)} \leq c \cdot h^{1 / 4} \cdot\|\delta q\|_{\mathrm{H}^{2}(I)}^{2}$.

Proof. As $q_{\sigma}$ as well as $\delta q$ are fixed throughout in this proof, we will use the following abbreviations.

$$
\begin{aligned}
u & =S\left(q_{\sigma}\right), & u_{h} & =S_{h}\left(q_{\sigma}\right), \\
\delta u & =S^{\prime}\left(q_{\sigma}\right)(\delta q), & \delta u_{h} & =S_{h}^{\prime}\left(q_{\sigma}\right)(\delta q), \\
\tau u & =S^{\prime \prime}\left(q_{\sigma}\right)(\delta q, \delta q), & \tau u_{h} & =S_{h}^{\prime \prime}\left(q_{\sigma}\right)(\delta q, \delta q) .
\end{aligned}
$$

(1) As $u_{h}$ is just the finite-element approximation of $u \in \mathrm{H}^{2}\left(\Omega_{0}\right)$, i.e.

$$
\begin{aligned}
a\left(q_{\sigma}\right)(u, v) & =l\left(q_{\sigma}\right)(v) & & \forall v \in \mathrm{H}_{0}^{1}\left(\Omega_{q}\right), \\
a\left(q_{\sigma}\right)\left(u_{h}, v_{h}\right) & =l\left(q_{\sigma}\right)\left(v_{h}\right) & & \forall v_{h} \in V_{h},
\end{aligned}
$$

we can use standard estimation techniques to proof the first assertion.

(2) For $v \in \mathrm{H}_{0}^{1}\left(\Omega_{0}\right)$, let the functional $F \in \mathrm{H}^{-1}\left(\Omega_{0}\right)$ be defined as

$$
F(v)=\left(\nabla f \circ T_{q_{\sigma}} \cdot V_{\delta q}, v \gamma_{q_{\sigma}}\right)+\left(f \circ T_{q_{\sigma}}, v \operatorname{div}\left(V_{\delta q}\right)\right)-\left(u, v \operatorname{div}\left(V_{\delta q}\right)\right)+\left(\operatorname{div}\left(A_{q_{\sigma}, \delta q}^{\prime} \cdot \nabla u\right), v\right) .
$$

Then, by definition of $\delta u$, it holds that

$$
a\left(q_{\sigma}\right)(\delta u, v)=F(v) \quad \forall v \in \mathrm{H}_{0}^{1}\left(\Omega_{0}\right) .
$$

We want to use Theorem 1.12, part (1), with $s=1 / 4$, so we have to find bounds for $\|F\|_{\mathrm{H}^{-3 / 4}\left(\Omega_{0}\right)}$. In the next steps we make use of the continuous embeddings $\mathrm{L}^{2}\left(\Omega_{0}\right), \mathrm{L}^{3 / 2}\left(\Omega_{0}\right) \hookrightarrow \mathrm{H}^{-3 / 4}\left(\Omega_{0}\right)$, Corollary 3.34 and the generalized Hölder's inequality.

$$
\begin{aligned}
\|F\|_{\mathrm{H}^{-3 / 4}\left(\Omega_{0}\right)} \leq & \left\|\nabla f \circ T_{q_{\sigma}}\right\|_{\mathrm{L}^{2}\left(\Omega_{0}\right)} \cdot\left\|V_{\delta q}\right\|_{\mathrm{L}^{\infty}\left(\Omega_{0}\right)} \cdot\left\|\gamma_{q_{\sigma}}\right\|_{\mathrm{L}^{\infty}\left(\Omega_{0}\right)}+\left\|f \circ T_{q_{\sigma}}\right\|_{\mathrm{L}^{2}\left(\Omega_{0}\right)} \cdot\left\|\operatorname{div}\left(V_{\delta q}\right)\right\|_{\mathrm{L}^{\infty}\left(\Omega_{0}\right)} \\
& +\|u\|_{\mathrm{L}^{2}\left(\Omega_{0}\right)} \cdot\left\|\operatorname{div}\left(V_{\delta q}\right)\right\|_{\mathrm{L}^{\infty}\left(\Omega_{0}\right)}+\left\|A_{q_{\sigma}, \delta q}^{\prime}\right\|_{\mathrm{L}^{\infty}\left(\Omega_{0}\right)} \cdot\|u\|_{\mathrm{H}^{2}\left(\Omega_{0}\right)} \\
& +\left\|\operatorname{div}\left(A_{q_{\sigma}, \delta q}^{\prime}\right)\right\|_{\mathrm{L}^{2}\left(\Omega_{0}\right)} \cdot\|u\|_{\mathrm{W}^{1,6}\left(\Omega_{0}\right)} \cdot
\end{aligned}
$$


Now we use the Lemmata 1.6 and 1.7, Corollary 3.38 and the embedding $\mathrm{H}^{2}\left(\Omega_{0}\right) \hookrightarrow \mathrm{W}^{1,6}\left(\Omega_{0}\right)$ and arrive at

$$
\|F\|_{\mathrm{H}^{-3 / 4}\left(\Omega_{0}\right)} \leq c \cdot\|\delta q\|_{\mathrm{H}^{2}(I)} .
$$

As a result we can now make use of Theorem 1.12, part (1). Together with Lemma 3.6 this implies $\delta u \in \mathrm{H}^{5 / 4}\left(\Omega_{0}\right)$ and

$$
\|\delta u\|_{\mathrm{H}^{5 / 4}\left(\Omega_{0}\right)} \leq c \cdot\|\delta q\|_{\mathrm{H}^{2}(I)},
$$

where the constant $c$ is independent of $q_{\sigma}$ and $\delta q$. As $\mathrm{H}^{5 / 4}\left(\Omega_{0}\right) \hookrightarrow \mathrm{C}^{0}\left(\bar{\Omega}_{0}\right)$, the pointwise interpolation $i_{h} \delta u \in V_{h}$ of $\delta u$ is well defined. Now it holds that

$$
\begin{aligned}
c_{1} \cdot\left\|i_{h} \delta u-\delta u_{h}\right\|_{\mathrm{H}^{1}\left(\Omega_{0}\right)}^{2} \leq & a\left(q_{\sigma}\right)\left(i_{h} \delta u-\delta u_{h}, i_{h} \delta u-\delta u_{h}\right) \\
= & a\left(q_{\sigma}\right)\left(\delta u-\delta u_{h}, i_{h} \delta u-\delta u_{h}\right)-a\left(q_{\sigma}\right)\left(\delta u-i_{h} \delta u, i_{h} \delta u-\delta u_{h}\right) \\
= & \left(\nabla\left(u-u_{h}\right), A_{q_{\sigma}, \delta q}^{\prime} \cdot \nabla\left(i_{h} \delta u-\delta u_{h}\right)\right)+\left(u-u_{h},\left(i_{h} \delta u-\delta u_{h}\right) \operatorname{div}\left(V_{\delta q}\right)\right) \\
& -a\left(q_{\sigma}\right)\left(\delta u-i_{h} \delta u, i_{h} \delta u-\delta u_{h}\right) \\
\leq & c_{2} \cdot h \cdot\left\|i_{h} \delta u-\delta u_{h}\right\|_{\mathrm{H}^{1}\left(\Omega_{0}\right)} \cdot\|\delta q\|_{\mathrm{H}^{2}(I)}+c_{3} \cdot\left\|\delta u-i_{h} \delta u\right\|_{\mathrm{H}^{1}\left(\Omega_{0}\right)} \cdot\left\|i_{h} \delta u-\delta u_{h}\right\|_{\mathrm{H}^{1}\left(\Omega_{0}\right)},
\end{aligned}
$$

where we made use of part (1) of this lemma. Using Young's inequality, for $\varepsilon>0$ we get

$$
\left(c_{1}-\varepsilon\right) \cdot\left\|i_{h} \delta u-\delta u_{h}\right\|_{\mathrm{H}^{1}\left(\Omega_{0}\right)}^{2} \leq \frac{c_{2}^{2}}{2 \cdot \varepsilon} \cdot h^{2} \cdot\|\delta q\|_{\mathrm{H}^{2}(I)}^{2}+\frac{c_{3}^{2}}{2 \cdot \varepsilon} \cdot\left\|\delta u-i_{h} \delta u\right\|_{\mathrm{H}^{1}\left(\Omega_{0}\right)}^{2},
$$

where we now choose $\varepsilon=c_{1} / 2$. Using interpolation estimates as shown in [10] it now holds that

$$
\left\|\delta u-i_{h} \delta u\right\|_{\mathrm{H}^{1}\left(\Omega_{0}\right)} \leq c \cdot h^{1 / 4} \cdot\|\delta u\|_{\mathrm{H}^{5 / 4}\left(\Omega_{0}\right)} \leq c \cdot h^{1 / 4} \cdot\|\delta q\|_{\mathrm{H}^{2}(I)} .
$$

Combining (3.29) and (3.30) we end up with

$$
\begin{aligned}
\left\|\delta u-\delta u_{h}\right\|_{\mathrm{H}^{1}\left(\Omega_{0}\right)}^{2} & \leq 2 \cdot\left(\left\|\delta u-i_{h} \delta u\right\|_{\mathrm{H}^{1}\left(\Omega_{0}\right)}^{2}+\left\|i_{h} \delta u-\delta u_{h}\right\|_{\mathrm{H}^{1}\left(\Omega_{0}\right)}^{2}\right) \\
& \leq c \cdot h^{1 / 2} \cdot\|\delta q\|_{\mathrm{H}^{2}(I)}^{2},
\end{aligned}
$$

and finish the proof by taking the square root on both sides.

(3) First of all we introduce kind of an "intermediate" derivative $\tau \tilde{u}_{h}$.

Definition 3.36. Let $\tau \tilde{u}_{h} \in V_{h}$ be the solution of

$$
\begin{aligned}
a\left(q_{\sigma}\right)\left(\tau \tilde{u}_{h}, v_{h}\right)= & \left(V_{\delta q}^{T} \cdot \nabla^{2} f \circ T_{q_{\sigma}} \cdot V_{\delta q}, v_{h} \gamma_{q_{\sigma}}\right)+2 \times\left(\left(\nabla f \circ T_{q_{\sigma}}\right) \cdot V_{\delta q}, v_{h} \operatorname{div}\left(V_{\delta q}\right)\right) \\
& -\left(\nabla u, A_{q_{\sigma}, \delta q, \delta q}^{\prime \prime} \nabla v_{h}\right)-2 \times\left(\nabla \delta u, A_{q_{\sigma}, \delta q}^{\prime} \nabla v_{h}\right)-\left(\delta u, v_{h} \operatorname{div}\left(V_{\delta q}\right)\right) \quad \forall v_{h} \in V_{h} .
\end{aligned}
$$

Next we split

$$
\left\|\tau u-\tau u_{h}\right\|_{L^{2}\left(\Omega_{0}\right)} \leq\|\underbrace{\tau u-\tau \tilde{u}_{h}}_{=e_{1}}\|_{L^{2}\left(\Omega_{0}\right)}+\|\underbrace{\tau \tilde{u}_{h}-\tau u_{h}}_{=e_{2}}\|_{L^{2}\left(\Omega_{0}\right)},
$$

and estimate both parts on the right hand side separately. 


\section{Estimation of $e_{2}$}

We start with the estimation of the second part. As $e_{2} \in V_{h}$, we get from Definition 3.36

$$
\begin{aligned}
c \cdot\left\|e_{2}\right\|_{\mathrm{H}^{1}\left(\Omega_{0}\right) \leq}^{2} \leq & a\left(q_{\sigma}\right)\left(e_{2}, e_{2}\right) \\
= & -\left(\nabla\left(u-u_{h}\right), A_{q_{\sigma}, \delta q, \delta q}^{\prime \prime} \cdot \nabla e_{2}\right)-2 \cdot\left(\nabla\left(\delta u-\delta u_{h}\right), A_{q_{\sigma}, \delta q}^{\prime} \cdot \nabla e_{2}\right)-\left(\delta u-\delta u_{h}, e_{2} \operatorname{div}\left(V_{\delta q}\right)\right) \\
\leq & \left\|u-u_{h}\right\|_{\mathrm{H}^{1}\left(\Omega_{0}\right)} \cdot\left\|A_{q_{\sigma}, \delta q, \delta q}^{\prime \prime}\right\|_{\mathrm{L}^{\infty}\left(\Omega_{0}\right)} \cdot\left\|e_{2}\right\|_{\mathrm{H}^{1}\left(\Omega_{0}\right)} \\
& +2 \cdot\left\|\delta u-\delta u_{h}\right\|_{\mathrm{H}^{1}\left(\Omega_{0}\right)} \cdot\left\|A_{q_{\sigma}, \delta q}^{\prime}\right\|_{\mathrm{L}^{\infty}\left(\Omega_{0}\right)} \cdot\left\|e_{2}\right\|_{\mathrm{H}^{1}\left(\Omega_{0}\right)} \\
& \quad+\left\|\delta u-\delta u_{h}\right\|_{\mathrm{L}^{2}\left(\Omega_{0}\right)} \cdot\left\|\operatorname{div}\left(V_{\delta q}\right)\right\|_{\mathrm{L}^{\infty}\left(\Omega_{0}\right)} \cdot\left\|e_{2}\right\|_{\mathrm{L}^{2}\left(\Omega_{0}\right)},
\end{aligned}
$$

and by using the first two parts of this lemma, as well as the Lemmata 1.6 and 1.7 we get

$$
\left\|e_{2}\right\|_{\mathrm{H}^{1}\left(\Omega_{0}\right)} \leq c \cdot h^{1 / 4} \cdot\|\delta q\|_{\mathrm{H}^{2}(I)}^{2} .
$$

Estimation of $e_{1}$

As $\tau \tilde{u}_{h}$ is just the Ritz-projection of $\tau u$, we have

$$
a\left(q_{\sigma}\right)\left(e_{1}, v_{h}\right)=0 \quad \forall v_{h} \in V_{h} .
$$

With a duality argument one deduces

$$
\left\|e_{1}\right\|_{\mathrm{L}^{2}\left(\Omega_{0}\right)} \leq c \cdot h \cdot\left\|e_{1}\right\|_{\mathrm{H}^{1}\left(\Omega_{0}\right)} .
$$

We continue with

$$
\left\|e_{1}\right\|_{\mathrm{H}^{1}\left(\Omega_{0}\right)} \leq\|\tau u\|_{\mathrm{H}^{1}\left(\Omega_{0}\right)}+\left\|\tau \tilde{u}_{h}\right\|_{\mathrm{H}^{1}\left(\Omega_{0}\right)} .
$$

The first part can be estimated using Lemma 3.6, a similar bound for the second part can be proven in the same way, which leads to

$$
\left\|e_{1}\right\|_{\mathrm{H}^{1}\left(\Omega_{0}\right)} \leq c \cdot\|\delta q\|_{\mathrm{H}^{2}(I)}^{2} .
$$

Collecting the Estimates (3.32), (3.33) and (3.34) finishes this proof.

In what follows we will also need estimates for the $\mathrm{W}^{2, p}\left(\Omega_{0}\right)$ norm of the states corresponding to controls from the discretized control space for a $p>2$. Due to Theorem 1.12, (4), we therefore have to ensure that the $\mathrm{W}^{2, \infty}(I)$ norm of the associated controls is bounded.

Definition 3.37. For a given $d>0$ let

$$
Q_{\sigma, d}^{a d}=\left\{q_{\sigma} \in Q_{\sigma}^{a d} \mid\left\|q_{\sigma}\right\|_{\mathrm{W}^{2, \infty}(I)} \leq d\right\} .
$$

Corollary 3.38. Let for a given $d>0$ be $\left\{q_{\sigma}\right\}_{\sigma>0}$ an arbitrary sequence of controls with $q_{\sigma} \in Q_{\sigma, d}^{a d}$ for $\sigma>0$. Then there exists $p_{\Omega_{0}}>2$ and it holds that $S\left(q_{\sigma}\right) \in \mathrm{W}^{2, p}\left(\Omega_{0}\right)$ for $p<p_{\Omega_{0}}$ and $\left\|S\left(q_{\sigma}\right)\right\|_{\mathrm{W}^{2, p}\left(\Omega_{0}\right)}$ is bounded independent of $\sigma$.

Proof. Due to Theorem 1.12, (4), we know that there exists $p_{\Omega_{0}}>2$ such that for all $p<p_{\Omega_{0}}$ it holds that

$$
\left\|S\left(q_{\sigma}\right)\right\|_{\mathrm{W}^{2, p}\left(\Omega_{0}\right)} \leq c \cdot\left\|f \circ T_{q} \gamma_{q}\right\|_{\mathrm{L}^{p}\left(\Omega_{0}\right)} .
$$

The constant $c$ can be bounded from above due to the boundedness of $q_{\sigma}$ in $\mathrm{W}^{2, \infty}(I)$. As $f$ was assumed to be sufficiently regular, $\gamma_{q}$ is bounded in $\mathrm{L}^{\infty}$ and $\hat{\Omega} \supset \Omega_{q}$ is bounded. It follows that the right hand side can be bounded from above.

Using boundedness of $q_{\sigma}$ in $\mathrm{W}^{2, \infty}(I)$ one obtains better estimates for the error between $S^{\prime}\left(q_{\sigma}\right)$ and $S_{h}^{\prime}\left(q_{\sigma}\right)$. 
Lemma 3.39. For $q_{\sigma} \in Q_{\sigma, d}^{a d}, \delta q \in Q$ and $k \in\{0,1\}$ it holds that

$$
\left\|S^{\prime}\left(q_{\sigma}\right)(\delta q)-S_{h}^{\prime}\left(q_{\sigma}\right)(\delta q)\right\|_{\mathrm{H}^{1-k}\left(\Omega_{0}\right)} \leq c \cdot h^{1+k} \cdot\|\delta q\|_{\mathrm{H}^{2}(I)} .
$$

Proof. As $q_{\sigma}$ as well as $\delta q$ are fixed throughout in this part, we will use the following abbreviations.

$$
\begin{aligned}
u & =S\left(q_{\sigma}\right), & u_{h} & =S_{h}\left(q_{\sigma}\right), \\
\delta u & =S^{\prime}\left(q_{\sigma}\right)(\delta q), & \delta u_{h} & =S_{h}^{\prime}\left(q_{\sigma}\right)(\delta q) .
\end{aligned}
$$

Additionally we introduce kind of an "intermediate" derivative $\delta \tilde{u}_{h}$.

Definition 3.40. Let $\delta \tilde{u}_{h} \in V_{h}$ be the solution of

$$
\begin{aligned}
a\left(q_{\sigma}\right)\left(\delta \tilde{u}_{h}, v_{h}\right)= & \left(\nabla f \circ T_{q_{\sigma}} \cdot V_{\delta q}, v_{h} \gamma_{q_{\sigma}}\right)+\left(f \circ T_{q_{\sigma}}, v_{h} \operatorname{div}\left(V_{\delta q}\right)\right) \\
& -\left(\nabla u \cdot A_{q_{\sigma}, \delta q}^{\prime}, \nabla v_{h}\right)-\left(u, v_{h} \operatorname{div}\left(V_{\delta q}\right)\right) \quad \forall v_{h} \in V_{h} .
\end{aligned}
$$

Now we split

$$
\left\|\delta u-\delta u_{h}\right\|_{\mathrm{H}^{1-k}\left(\Omega_{0}\right)} \leq\|\underbrace{\delta u-\delta \tilde{u}_{h}}_{=e_{1}}\|_{\mathrm{H}^{1-k}\left(\Omega_{0}\right)}+\|\underbrace{\delta \tilde{u}_{h}-\delta u_{h}}_{=e_{2}}\|_{\mathrm{H}^{1-k}\left(\Omega_{0}\right)},
$$

and estimate both parts on the right hand side separately.

\section{Estimation of $e_{2}$}

We start with the estimation for the latter part. From the definition of $e_{2}$ we get

$$
a\left(q_{\sigma}\right)\left(e_{2}, v_{h}\right)=\left(\nabla\left(u_{h}-u\right) \cdot A_{q_{\sigma}, \delta q}^{\prime}, \nabla v_{h}\right)+\left(u_{h}-u, v \operatorname{div}\left(V_{\delta q}\right)\right) \quad \forall v_{h} \in V_{h},
$$

and by choosing $v_{h}=e_{2}$,

$$
\begin{aligned}
a\left(q_{\sigma}\right)\left(e_{2}, e_{2}\right)= & \left.\left(\nabla\left(u_{h}-u\right) \cdot A_{q_{\sigma}, \delta q}^{\prime}, \nabla e_{2}\right)\right)+\left(u_{h}-u, e_{2} \operatorname{div}\left(V_{\delta q}\right)\right) \\
\leq & \left\|\nabla\left(u_{h}-u\right)\right\|_{L^{2}\left(\Omega_{0}\right)} \cdot\left\|A_{q_{\sigma}, \delta q}^{\prime}\right\|_{L^{\infty}\left(\Omega_{0}\right)} \cdot\left\|\nabla e_{2}\right\|_{L^{2}\left(\Omega_{0}\right)} \\
& +\left\|u_{h}-u\right\|_{L^{2}\left(\Omega_{0}\right)} \cdot\left\|\operatorname{div}\left(V_{\delta q}\right)\right\|_{L^{\infty}\left(\Omega_{0}\right)} \cdot\left\|e_{2}\right\|_{L^{2}\left(\Omega_{0}\right)} .
\end{aligned}
$$

This finally leads to

$$
\left\|e_{2}\right\|_{\mathrm{H}^{1}\left(\Omega_{0}\right)} \leq c \cdot h \cdot\|\delta q\|_{\mathrm{H}^{2}(I)}
$$

where we used the fact that $\left\|A_{q_{\sigma}, \delta q}^{\prime}\right\|_{L^{\infty}\left(\Omega_{0}\right)}$ and $\left\|\operatorname{div}\left(V_{\delta q}\right)\right\|_{L^{\infty}\left(\Omega_{0}\right)}$ are bounded by $c \cdot\|\delta q\|_{\mathrm{H}^{2}(I)}$, with $c$ being independent of $q_{\sigma}$ and $\sigma$.

Now we define $z, y \in \mathrm{H}_{0}^{1}\left(\Omega_{0}\right)$ and $z_{h} \in V_{h}$ as the solutions of

$$
\begin{aligned}
a\left(q_{\sigma}\right)(v, z) & =\left(e_{2}, v\right) & & \forall v \in \mathrm{H}_{0}^{1}\left(\Omega_{0}\right), \\
a\left(q_{\sigma}\right)\left(v_{h}, z_{h}\right) & =\left(e_{2}, v_{h}\right) & & \forall v_{h} \in V_{h}, \\
a\left(q_{\sigma}\right)(v, y) & =\left(\nabla z \cdot A_{q_{\sigma}, \delta q}^{\prime}, \nabla v\right)=-\left(\operatorname{div}\left(A_{q_{\sigma}, \delta q}^{\prime} \cdot \nabla z\right), v\right) & & \forall v \in \mathrm{H}_{0}^{1}\left(\Omega_{0}\right) .
\end{aligned}
$$

Theorem 1.12, (3), tells us that $z \in \mathrm{H}^{2}\left(\Omega_{0}\right)$ and $\|z\|_{\mathrm{H}^{2}\left(\Omega_{0}\right)} \leq c \cdot\left\|e_{2}\right\|_{\mathrm{L}^{2}\left(\Omega_{0}\right)}$. It now holds that

$$
\begin{aligned}
\left\|e_{2}\right\|_{L^{2}\left(\Omega_{0}\right)}^{2}= & a\left(q_{\sigma}\right)\left(e_{2}, z_{h}\right) \\
= & -\left(\nabla\left(u-u_{h}\right) \cdot A_{q_{\sigma}, \delta q}^{\prime}, \nabla z_{h}\right)-\left(u-u_{h}, z_{h} \operatorname{div}\left(V_{\delta q}\right)\right) \\
= & -\left(\nabla\left(u-u_{h}\right) \cdot A_{q_{\sigma}, \delta q}^{\prime}, \nabla z\right)+\left(\nabla\left(u-u_{h}\right) \cdot A_{q_{\sigma}, \delta q}^{\prime}, \nabla\left(z-z_{h}\right)\right) \\
& -\left(u-u_{h}, z \operatorname{div}\left(V_{\delta q}\right)\right)+\left(u-u_{h},\left(z-z_{h}\right) \operatorname{div}\left(V_{\delta q}\right)\right) .
\end{aligned}
$$


With the Lemmata 1.6, 1.7, 3.35 and standard finite-element error estimates we now have

$$
\begin{array}{r}
\left(\nabla\left(u-u_{h}\right) \cdot A_{q_{\sigma}, \delta q}^{\prime}, \nabla\left(z-z_{h}\right)\right) \leq c \cdot h^{2} \cdot\|\delta q\|_{\mathrm{H}^{2}(I)} \cdot\left\|e_{2}\right\|_{\mathrm{L}^{2}\left(\Omega_{0}\right)}, \\
\left(u-u_{h}, z \operatorname{div}\left(V_{\delta q}\right)\right) \leq c \cdot h^{2} \cdot\|\delta q\|_{\mathrm{H}^{2}(I)} \cdot\left\|e_{2}\right\|_{\mathrm{L}^{2}\left(\Omega_{0}\right)}, \\
\left(u-u_{h},\left(z-z_{h}\right) \operatorname{div}\left(V_{\delta q}\right)\right) \leq c \cdot h^{4} \cdot\|\delta q\|_{\mathrm{H}^{2}(I)} \cdot\left\|e_{2}\right\|_{\mathrm{L}^{2}\left(\Omega_{0}\right)} .
\end{array}
$$

Hence it remains to find some bounds for

$$
\begin{aligned}
\left(\nabla\left(u-u_{h}\right) \cdot A_{q_{\sigma}, \delta q}^{\prime}, \nabla z\right) & =a\left(q_{\sigma}\right)\left(u-u_{h}, y\right) \\
& =a\left(q_{\sigma}\right)\left(u-u_{h}, y-i_{h} y\right)+\underbrace{a\left(q_{\sigma}\right)\left(u-u_{h}, i_{h} y\right)}_{=0} \\
& \leq c \cdot\left\|y-i_{h} y\right\|_{\mathrm{W}^{1, \bar{p}^{\prime}}\left(\Omega_{0}\right)} \cdot\left\|u-u_{h}\right\|_{\mathrm{W}^{1, \bar{p}}\left(\Omega_{0}\right)},
\end{aligned}
$$

where we chose $\bar{p} \in\left(2, p_{\Omega_{0}}\right)$ with $p_{\Omega_{0}}>2$ as the constant from Theorem 1.12, part (4), and $\bar{p}+\bar{p}^{\prime-1}=1$. Here, $i_{h} y \in V_{h}$ shall denote the pointwise interpolation of $y$ which is well-defined according to (3.40). With Corollary 3.38 and a stability result for the $\mathrm{W}^{1, \bar{p}}\left(\Omega_{0}\right)$-error for the Ritz projection from [23] we get

$$
\left\|u-u_{h}\right\|_{\mathrm{W}^{1, \bar{p}}\left(\Omega_{0}\right)} \leq c \cdot h \cdot\|u\|_{\mathrm{W}^{2, \bar{p}}\left(\Omega_{0}\right)} \leq c \cdot h .
$$

To continue, we need some information about the regularity of $y$. From the symmetry of $A_{q_{\sigma}, \delta q}^{\prime}$ it now follows, together with Corollary 3.34,

$$
\operatorname{div}\left(A_{q_{\sigma}, \delta q}^{\prime} \cdot \nabla z\right)=A_{q_{\sigma}, \delta q}^{\prime} \bullet \nabla^{2} z+\operatorname{div}\left(A_{q_{\sigma}, \delta q}^{\prime}\right)^{T} \cdot \nabla z \in \mathrm{L}^{\bar{p}^{\prime}}\left(\Omega_{0}\right),
$$

where we also used $\bar{p}>2>\bar{p}^{\prime}, \mathrm{H}^{2}\left(\Omega_{0}\right) \hookrightarrow \mathrm{W}^{1, p}\left(\Omega_{0}\right)$ for $p<\infty$ as well as $\operatorname{div}\left(A_{q_{\sigma}, \delta q}^{\prime}\right) \in \mathrm{L}^{2}\left(\Omega_{0}\right)$, which can be obtained by plugging in the corresponding definition and the generalized Hölder's inequality. We can also estimate the norm

$$
\begin{aligned}
\left\|\operatorname{div}\left(A_{q_{\sigma}, \delta q}^{\prime} \cdot \nabla z\right)\right\|_{L_{\bar{p}^{\prime}}\left(\Omega_{0}\right)} & \leq c \cdot\left(\left\|A_{q_{\sigma}, \delta q}^{\prime} \bullet \nabla^{2} z\right\|_{\mathrm{L}^{\bar{p}^{\prime}}\left(\Omega_{0}\right)}+\left\|\operatorname{div}\left(A_{q_{\sigma}, \delta q}^{\prime}\right) \cdot \nabla z\right\|_{\mathrm{L}^{\bar{p}^{\prime}}\left(\Omega_{0}\right)}\right) \\
& \leq c \cdot\left(\left\|A_{q_{\sigma}, \delta q}^{\prime}\right\|_{\mathrm{L}^{\infty}\left(\Omega_{0}\right)} \cdot\left\|\nabla^{2} z\right\|_{\mathrm{L}^{\bar{p}^{\prime}}\left(\Omega_{0}\right)}+\left\|\operatorname{div}\left(A_{q_{\sigma}, \delta q}^{\prime}\right)\right\|_{\mathrm{L}^{2}\left(\Omega_{0}\right)} \cdot\|\nabla z\|_{\mathrm{L}^{\frac{2 \bar{p}^{\prime}}{2-\bar{p}^{\prime}}\left(\Omega_{0}\right)}}\right) \\
& \leq c \cdot\left(\left\|A_{q_{\sigma}, \delta q}^{\prime}\right\|_{\mathrm{L}^{\infty}\left(\Omega_{0}\right)}+\left\|\operatorname{div}\left(A_{q_{\sigma}, \delta q}^{\prime}\right)\right\|_{\mathrm{L}^{2}\left(\Omega_{0}\right)}\right) \cdot\|z\|_{\mathrm{H}^{2}\left(\Omega_{0}\right)} \\
& \leq c \cdot\|\delta q\|_{\mathrm{H}^{2}(I)} \cdot\left\|e_{2}\right\|_{\mathrm{L}^{2}\left(\Omega_{0}\right)},
\end{aligned}
$$

where the constant $c$ just depends on $\bar{p}$, for $\Omega_{0}$ is fixed. Now we can again apply Corollary 3.38 to obtain

$$
y \in \mathrm{W}^{2, \bar{p}^{\prime}}\left(\Omega_{0}\right) \hookrightarrow \mathrm{C}^{0}\left(\bar{\Omega}_{0}\right) \text { for } \bar{p}^{\prime}>1,
$$

which results in

$$
\|y\|_{\mathrm{W}^{2, \bar{p}^{\prime}}\left(\Omega_{0}\right)} \leq c \cdot\left\|\operatorname{div}\left(A_{q_{\sigma}, \delta q}^{\prime} \cdot \nabla z\right)\right\|_{\mathrm{L}^{\bar{p}^{\prime}}\left(\Omega_{0}\right)} \leq c \cdot\|\delta q\|_{\mathrm{H}^{2}(I)} \cdot\left\|e_{2}\right\|_{\mathrm{L}^{2}\left(\Omega_{0}\right)} .
$$

Now we can apply standard interpolation results [23] and get

$$
\left\|y-i_{h} y\right\|_{\mathrm{W}^{1, \bar{p}^{\prime}}\left(\Omega_{0}\right)} \leq c \cdot h \cdot\|y\|_{\mathrm{W}^{2, \bar{p}^{\prime}}\left(\Omega_{0}\right)} \leq c \cdot h \cdot\|\delta q\|_{\mathrm{H}^{2}(I)} \cdot\left\|e_{2}\right\|_{\mathrm{L}^{2}\left(\Omega_{0}\right)} .
$$

Collecting the Estimates (3.38), (3.39) and (3.42) finally leads to

$$
\left\|e_{2}\right\|_{\mathrm{L}^{2}\left(\Omega_{0}\right)} \leq c \cdot h^{2} \cdot\|\delta q\|_{\mathrm{H}^{2}(I)} .
$$




\section{Estimation of $e_{1}$}

To estimate the first part we proceed similar to the latter one. Because of

$$
-\left(\nabla u \cdot A_{q_{\sigma}, \delta q}^{\prime}, \nabla v\right)=\left(\operatorname{div}\left(A_{q_{\sigma}, \delta q}^{\prime} \cdot \nabla u\right), v\right) \quad \forall v \in \mathrm{H}_{0}^{1}\left(\Omega_{0}\right),
$$

and $u \in \mathrm{W}^{2, \bar{p}}\left(\Omega_{0}\right) \hookrightarrow \mathrm{W}^{1, \infty}\left(\Omega_{0}\right)$, we again derive, similar to $(3.41)$, that $\delta u \in \mathrm{H}^{2}\left(\Omega_{0}\right),\|\delta u\|_{\mathrm{H}^{2}\left(\Omega_{0}\right)} \leq c \cdot\|\delta q\|_{\mathrm{H}^{2}(I)}$, where we also used the fact that $\left\|f \circ T_{q_{\sigma}}\right\|_{L^{p}\left(\Omega_{0}\right)}$ is bounded independently of $p \in[1, \infty]$. As $e_{1}$ is just the error of the Ritz-projection for $\delta u$, we immediately get

$$
\left\|e_{1}\right\|_{\mathrm{H}^{1}\left(\Omega_{0}\right)} \leq c \cdot h \cdot\|\delta q\|_{\mathrm{H}^{2}(I)},
$$

and with a duality argument it follows that

$$
\left\|e_{1}\right\|_{\mathrm{L}^{2}\left(\Omega_{0}\right)} \leq c \cdot h \cdot\left\|e_{1}\right\|_{\mathrm{H}^{1}\left(\Omega_{0}\right)} .
$$

Combining the estimates (3.44) and (3.45) leads to

$$
\left\|e_{1}\right\|_{\mathrm{L}^{2}\left(\Omega_{0}\right)} \leq c \cdot h^{2} \cdot\|\delta q\|_{\mathrm{H}^{2}(I)} .
$$

Adding (3.37) and (3.44) gives the estimation for the $\mathrm{H}^{1}$-error, by adding (3.43) and (3.46) it is possible to estimate the $\mathrm{L}^{2}$-error.

Lemma 3.41. For $q_{\sigma} \in Q_{\sigma}^{a d}, \delta q \in Q$ it holds that

1. $\left|j\left(q_{\sigma}\right)-j_{h}\left(q_{\sigma}\right)\right| \leq c \cdot h^{2}$,

2. $\left|j^{\prime \prime}\left(q_{\sigma}\right)(\delta q, \delta q)-j_{h}^{\prime \prime}\left(q_{\sigma}\right)(\delta q, \delta q)\right| \leq c \cdot h^{1 / 4} \cdot\|\delta q\|_{\mathrm{H}^{2}(I)}^{2}$.

Proof. (1) We have

$$
\begin{aligned}
\left|j\left(q_{\sigma}\right)-j_{h}\left(q_{\sigma}\right)\right| & =\frac{1}{2}\left|\left(S\left(q_{\sigma}\right)-u_{d} \circ T_{q_{\sigma}},\left(S\left(q_{\sigma}\right)-u_{d} \circ T_{q_{\sigma}}\right) \gamma_{q_{\sigma}}\right)-\left(S_{h}\left(q_{\sigma}\right)-u_{d} \circ T_{q_{\sigma}},\left(S_{h}\left(q_{\sigma}\right)-u_{d} \circ T_{q_{\sigma}}\right) \gamma_{q_{\sigma}}\right)\right| \\
& =\frac{1}{2}\left|\left(S\left(q_{\sigma}\right)-S_{h}\left(q_{\sigma}\right),\left(S\left(q_{\sigma}\right)+S_{h}\left(q_{\sigma}\right)-2 \cdot u_{d} \circ T_{q_{\sigma}}\right) \gamma_{q_{\sigma}}\right)\right| .
\end{aligned}
$$

With Cauchy-Schwarz and the Lemmata 1.6, 3.6, 3.33 and 3.35 we arrive at

$$
\left|j\left(q_{\sigma}\right)-j_{h}\left(q_{\sigma}\right)\right| \leq c \cdot h^{2} .
$$

(2) All the terms that do not contain a second derivative of $S$ can be estimated just in the same way as done in the first part of this proof and in the proof of Lemma 3.42, we only have to find some bounds for

$$
\begin{aligned}
& \mid\left(S\left(q_{\sigma}\right)-u_{d} \circ T_{q_{\sigma}},\left(S^{\prime \prime}\left(q_{\sigma}\right)(\delta q, \delta q)-V_{\delta q}^{T} \cdot \nabla^{2} u_{d} \circ T_{q_{\sigma}} \cdot V_{\delta q}\right) \gamma_{q_{\sigma}}\right) \\
& -\left(S_{h}\left(q_{\sigma}\right)-u_{d} \circ T_{q_{\sigma}},\left(S_{h}^{\prime \prime}\left(q_{\sigma}\right)(\delta q, \delta q)-V_{\delta q}^{T} \cdot \nabla^{2} u_{d} \circ T_{q_{\sigma}} \cdot V_{\delta q}\right) \gamma_{q_{\sigma}}\right) \mid \\
\leq & \left|\left(S\left(q_{\sigma}\right)-S_{h}\left(q_{\sigma}\right),\left(S^{\prime \prime}\left(q_{\sigma}\right)(\delta q, \delta q)-V_{\delta q}^{T} \cdot \nabla^{2} u_{d} \circ T_{q_{\sigma}} \cdot V_{\delta q}\right) \gamma_{q_{\sigma}}\right)\right| \\
& +\left|\left(S_{h}\left(q_{\sigma}\right)-u_{d} \circ T_{q_{\sigma}},\left(S^{\prime \prime}\left(q_{\sigma}\right)(\delta q, \delta q)-S_{h}^{\prime \prime}\left(q_{\sigma}\right)(\delta q, \delta q)\right) \gamma_{q_{\sigma}}\right)\right| \\
\leq & \left\|S\left(q_{\sigma}\right)-S_{h}\left(q_{\sigma}\right)\right\|_{\mathrm{L}^{2}\left(\Omega_{0}\right)} \cdot\left(\left\|S^{\prime \prime}\left(q_{\sigma}\right)(\delta q, \delta q)\right\|_{\mathrm{L}^{2}\left(\Omega_{0}\right)}+\left\|V_{\delta q}^{T} \cdot \nabla^{2} u_{d} \circ T_{q_{\sigma}} \cdot V_{\delta q}\right\|_{\mathrm{L}^{2}\left(\Omega_{0}\right)}\right) \cdot\left\|\gamma_{q_{\sigma}}\right\|_{\mathrm{L}^{\infty}\left(\Omega_{0}\right)} \\
& +\left(\left\|S_{h}\left(q_{\sigma}\right)\right\|_{\mathrm{L}^{2}\left(\Omega_{0}\right)}+\left\|u_{d} \circ T_{q_{\sigma}}\right\|_{\mathrm{L}^{2}\left(\Omega_{0}\right)}\right) \cdot\left\|S^{\prime \prime}\left(q_{\sigma}\right)(\delta q, \delta q)-S_{h}^{\prime \prime}\left(q_{\sigma}\right)(\delta q, \delta q)\right\|_{\mathrm{L}^{2}\left(\Omega_{0}\right)} \cdot\left\|\gamma_{q_{\sigma}}\right\|_{\mathrm{L}^{\infty}\left(\Omega_{0}\right)}, \\
\leq & c \cdot h^{1 / 4} \cdot\|\delta q\|_{\mathrm{H}^{2}(I)}^{2} .
\end{aligned}
$$

In the last step we made use of Lemmata 1.6 and 3.39 . 
Lemma 3.42. For $q_{\sigma} \in Q_{\sigma, d}^{a d}, \delta q \in Q$ it holds that

$$
\left|j^{\prime}\left(q_{\sigma}\right)(\delta q)-j_{h}^{\prime}\left(q_{\sigma}\right)(\delta q)\right| \leq c \cdot h^{2} \cdot\|\delta q\|_{\mathrm{H}^{2}(I)} .
$$

Proof. With (3.8) we get

$$
\begin{aligned}
j^{\prime}\left(q_{\sigma}\right)(\delta q)-j_{h}^{\prime}\left(q_{\sigma}\right)(\delta q)= & \frac{1}{2}\left(S\left(q_{\sigma}\right)-u_{d} \circ T_{q_{\sigma}},\left(S\left(q_{\sigma}\right)-u_{d} \circ T_{q_{\sigma}}\right) \operatorname{div}\left(V_{\delta q}\right)\right) \\
& -\frac{1}{2}\left(S_{h}\left(q_{\sigma}\right)-u_{d} \circ T_{q_{\sigma}},\left(S_{h}\left(q_{\sigma}\right)-u_{d} \circ T_{q_{\sigma}}\right) \operatorname{div}\left(V_{\delta q}\right)\right) \\
& +\left(S^{\prime}\left(q_{\sigma}\right)(\delta q)-\left(\nabla u_{d} \circ T_{q_{\sigma}}\right)^{T} \cdot V_{\delta q},\left(S\left(q_{\sigma}\right)-u_{d} \circ T_{q_{\sigma}}\right) \gamma_{q_{\sigma}}\right) \\
& -\left(S_{h}^{\prime}\left(q_{\sigma}\right)(\delta q)-\left(\nabla u_{d} \circ T_{q_{\sigma}}\right)^{T} \cdot V_{\delta q},\left(S_{h}\left(q_{\sigma}\right)-u_{d} \circ T_{q_{\sigma}}\right) \gamma_{q_{\sigma}}\right) .
\end{aligned}
$$

Using the Lemmata 1.6, 3.6, 3.33, 3.35 and 3.39 we can estimate both the corresponding parts

$$
\begin{aligned}
& \left|\left(S\left(q_{\sigma}\right)-u_{d} \circ T_{q_{\sigma}},\left(S\left(q_{\sigma}\right)-u_{d} \circ T_{q_{\sigma}}\right) \operatorname{div}\left(V_{\delta q}\right)\right)-\left(S_{h}\left(q_{\sigma}\right)-u_{d} \circ T_{q_{\sigma}},\left(S_{h}\left(q_{\sigma}\right)-u_{d} \circ T_{q_{\sigma}}\right) \operatorname{div}\left(V_{\delta q}\right)\right)\right| \\
& =\left|\left(S\left(q_{\sigma}\right)-S_{h}\left(q_{\sigma}\right),\left(S\left(q_{\sigma}\right)+S_{h}\left(q_{\sigma}\right)-2 \cdot u_{d} \circ T_{q_{\sigma}}\right) \operatorname{div}\left(V_{\delta q}\right)\right)\right|, \\
& \leq c \cdot h^{2} \cdot\|\delta q\|_{\mathrm{H}^{2}(I)},
\end{aligned}
$$

and

$$
\begin{aligned}
& \mid\left(S^{\prime}\left(q_{\sigma}\right)(\delta q)-\left(\nabla u_{d} \circ T_{q_{\sigma}}\right)^{T} \cdot V_{\delta q},\left(S\left(q_{\sigma}\right)-u_{d} \circ T_{q_{\sigma}}\right) \gamma_{q_{\sigma}}\right) \\
& -\left(S_{h}^{\prime}\left(q_{\sigma}\right)(\delta q)-\left(\nabla u_{d} \circ T_{q_{\sigma}}\right)^{T} \cdot V_{\delta q},\left(S_{h}\left(q_{\sigma}\right)-u_{d} \circ T_{q_{\sigma}}\right) \gamma_{q_{\sigma}}\right) \mid \\
\leq & \left|\left(S^{\prime}\left(q_{\sigma}\right)(\delta q)-S_{h}^{\prime}\left(q_{\sigma}\right)(\delta q),\left(S\left(q_{\sigma}\right)-u_{d} \circ T_{q_{\sigma}}\right) \gamma_{q_{\sigma}}\right)\right| \\
& +\left|\left(S_{h}^{\prime}\left(q_{\sigma}\right)(\delta q)-\left(\nabla u_{d} \circ T_{q_{\sigma}}\right)^{T} \cdot V_{\delta q},\left(S\left(q_{\sigma}\right)-S_{h}\left(q_{\sigma}\right)\right) \gamma_{q_{\sigma}}\right)\right| \\
\leq & c \cdot\left\|S^{\prime}\left(q_{\sigma}\right)(\delta q)-S_{h}^{\prime}\left(q_{\sigma}\right)(\delta q)\right\|_{\mathrm{L}^{2}\left(\Omega_{0}\right)} \\
& +c \cdot\left(\left\|S_{h}^{\prime}\left(q_{\sigma}\right)(\delta q)\right\|_{\mathrm{L}^{2}\left(\Omega_{0}\right)}+\left\|\left(\nabla u_{d} \circ T_{q_{\sigma}}\right)^{T} \cdot V_{\delta q}\right\|_{\mathrm{L}^{2}\left(\Omega_{0}\right)}\right) \cdot\left\|S\left(q_{\sigma}\right)-S_{h}\left(q_{\sigma}\right)\right\|_{\mathrm{L}^{2}\left(\Omega_{0}\right)} \\
\leq & c \cdot h^{2} \cdot\|\delta q\|_{\mathrm{H}^{2}(I)}+c \cdot\|\delta q\|_{\mathrm{H}^{2}(I)} \cdot h^{2} \\
\leq & c \cdot h^{2} \cdot\|\delta q\|_{\mathrm{H}^{2}(I)} \cdot
\end{aligned}
$$

Adding these two inequalities finishes this proof.

As mentioned in (3.3), we now have to prove the following lemma.

Lemma 3.43. There exists a sequence $\left(\bar{q}_{\sigma, h}\right)_{\sigma, h>0}$ of local optimal solutions of (2.4) with

$$
\left\|\bar{q}-\bar{q}_{\sigma, h}\right\|_{\mathrm{H}^{2}(I)} \rightarrow 0 \quad \text { for }(\sigma, h) \rightarrow 0 .
$$

Again, we call $\bar{q}_{\sigma, h}$ local optimal for (2.4) if there exists $\varepsilon>0$ with

$$
j_{h}\left(\bar{q}_{\sigma, h}\right) \leq j_{h}\left(q_{\sigma, h}\right) \quad \forall q_{\sigma, h} \in Q_{\sigma}^{a d},\left\|q_{\sigma, h}-\bar{q}_{\sigma, h}\right\|_{\mathrm{H}^{2}(I)} \leq \varepsilon .
$$

The detailed proof of Lemma 3.43 is omitted for it is again based on the ideas presented in [6] and similar to the existence proof of the sequence $\left(\bar{q}_{\sigma}\right)_{\sigma>0}$ from the previous subsection. The first part is to show that for $\bar{q}_{\sigma}$ being a fixed local optimal solution of (2.3) with $\left\|\bar{q}-\bar{q}_{\sigma}\right\|_{\mathrm{H}^{2}(I)}$ and $\sigma$ sufficiently small there exists a sequence $\left(\tilde{q}_{\sigma, h}\right)_{h>0}$ of local optimal solutions of $(2.4)$ with $\left\|\bar{q}_{\sigma}-\tilde{q}_{\sigma, h}\right\|_{\mathrm{H}^{2}(I)} \rightarrow 0$ for $h \rightarrow 0$. The existence of $\left(\bar{q}_{\sigma, h}\right)_{\sigma, h>0}$ then follows by a diagonal argument.

Now we have to make sure that we can apply some of the previous lemmata to the sequence $\left(\bar{q}_{\sigma}\right)_{\sigma>0}$. 
Lemma 3.44. Let $\left(\bar{q}_{\sigma}\right)_{\sigma>0}$ be a sequence as defined in (3.20). Then there exists $d>0$ such that for all $\sigma>0$ it holds that $\bar{q}_{\sigma} \in Q_{\sigma, d}^{a d}$.

Proof. Let $i_{\sigma}$ be the interpolation operator according to Definition 3.26. We have to find a bound for

$$
\left\|\bar{q}_{\sigma}\right\|_{\mathrm{W}^{2, \infty}(I)} \leq\left\|\bar{q}_{\sigma}-i_{\sigma} \bar{q}\right\|_{\mathrm{W}^{2, \infty}(I)}+\left\|i_{\sigma} \bar{q}\right\|_{\mathrm{W}^{2, \infty}(I)} .
$$

The latter part is bounded, as $i_{\sigma} \bar{q}$ is piecewise a polynomial whose coefficients depend continuously on $\|\bar{q}\|_{\mathrm{C}^{1,1}(\bar{I})}$, which is bounded due to Theorem 3.16. For the first part we use an inverse estimate: there exists a constant $c$, independent of $\sigma$, with

$$
\left\|\bar{q}_{\sigma}-i_{\sigma} \bar{q}\right\|_{\mathrm{W}^{2, \infty}(I)} \leq c \cdot \sigma^{-1 / 2} \cdot\left\|\bar{q}_{\sigma}-i_{\sigma} \bar{q}\right\|_{\mathrm{H}^{2}(I)} \rightarrow 0
$$

for $\sigma \rightarrow 0$ as shown in (3.25).

Now we are able to finish the proof of Theorem 3.2 by estimating the state error. Again with the Mean Value Theorem and Lemma 3.14 we get for some $t \in[0,1]$ and $\xi=(1-t) \cdot \bar{q}_{\sigma}+t \cdot \bar{q}_{\sigma, h}$,

$$
\begin{aligned}
\frac{\beta}{2} \cdot\left\|\bar{q}_{\sigma}-\bar{q}_{\sigma, h}\right\|_{\mathrm{H}^{2}(I)}^{2} & \leq j^{\prime \prime}(\xi)\left(\bar{q}_{\sigma}-\bar{q}_{\sigma, h}, \bar{q}_{\sigma}-\bar{q}_{\sigma, h}\right) \\
& \leq j_{h}^{\prime \prime}(\xi)\left(\bar{q}_{\sigma}-\bar{q}_{\sigma, h}, \bar{q}_{\sigma}-\bar{q}_{\sigma, h}\right)+\left|j^{\prime \prime}(\xi)\left(\bar{q}_{\sigma}-\bar{q}_{\sigma, h}, \bar{q}_{\sigma}-\bar{q}_{\sigma, h}\right)-j_{h}^{\prime \prime}(\xi)\left(\bar{q}_{\sigma}-\bar{q}_{\sigma, h}, \bar{q}_{\sigma}-\bar{q}_{\sigma, h}\right)\right| .
\end{aligned}
$$

Now we apply Lemma 3.41 and get

$$
\begin{aligned}
\frac{\beta}{2} \cdot\left\|\bar{q}_{\sigma}-\bar{q}_{\sigma, h}\right\|_{\mathrm{H}^{2}(I)}^{2} & \leq\left|j_{h}^{\prime}\left(\bar{q}_{\sigma}\right)\left(\bar{q}_{\sigma}-\bar{q}_{\sigma, h}\right)-j_{h}^{\prime}\left(\bar{q}_{\sigma, h}\right)\left(\bar{q}_{\sigma}-\bar{q}_{\sigma, h}\right)\right|+c_{1} \cdot h^{1 / 4} \cdot\left\|\bar{q}_{\sigma}-\bar{q}_{\sigma, h}\right\|_{\mathrm{H}^{2}(I)}^{2} \\
& =\left|j_{h}^{\prime}\left(\bar{q}_{\sigma}\right)\left(\bar{q}_{\sigma}-\bar{q}_{\sigma, h}\right)-j^{\prime}\left(\bar{q}_{\sigma}\right)\left(\bar{q}_{\sigma}-\bar{q}_{\sigma, h}\right)\right|+c_{1} \cdot h^{1 / 4} \cdot\left\|\bar{q}_{\sigma}-\bar{q}_{\sigma, h}\right\|_{\mathrm{H}^{2}(I)}^{2}
\end{aligned}
$$

where we again used the fact that $j^{\prime}\left(\bar{q}_{\sigma}\right)\left(\bar{q}_{\sigma}-\bar{q}_{\sigma, h}\right)=j_{h}^{\prime}\left(\bar{q}_{\sigma, h}\right)\left(\bar{q}_{\sigma}-\bar{q}_{\sigma, h}\right)=0$ due to Corollary 3.31 and the first order optimality conditions for $\bar{q}_{\sigma, h}$. Now we apply Lemma 3.42 and exploit the boundedness of $Q^{a d}$ to finally obtain

$$
\left(\frac{\beta}{2}-c_{1} \cdot h^{1 / 4}\right) \cdot\left\|\bar{q}_{\sigma}-\bar{q}_{\sigma, h}\right\|_{\mathrm{H}^{2}(I)}^{2} \leq c_{2} \cdot h^{2} \cdot\left\|\bar{q}_{\sigma}-\bar{q}_{\sigma, h}\right\|_{\mathrm{H}^{2}(I)} .
$$

So, for $h$ sufficiently small, i.e.

$$
h \leq\left(\frac{\beta}{4 c_{1}}\right)^{4},
$$

we finally arrive at

$$
\left\|\bar{q}_{\sigma}-\bar{q}_{\sigma, h}\right\|_{\mathrm{H}^{2}(I)} \leq \frac{4 c_{2}}{\beta} \cdot h^{2} .
$$

Collecting the estimates (3.24), (3.25) and (3.47) finishes the proof of the main result, Theorem 3.2.

Having proved this theorem, one can also estimate the error between the optimal continuous state and its discrete counterpart.

Corollary 3.45. It holds that

1. $\left\|S(\bar{q})-S_{h}\left(\bar{q}_{\sigma, h}\right)\right\|_{\mathrm{L}^{2}\left(\Omega_{0}\right)}=\mathcal{O}\left(\sigma^{2}+h^{2}\right)$,

2. $\left\|\tilde{S}(\bar{q}) \circ T_{\bar{q}}^{-1}-\tilde{S}_{h}\left(\bar{q}_{\sigma, h}\right) \circ T_{\bar{q}_{\sigma, h}}^{-1}\right\|_{L^{2}(\hat{\Omega})}=\mathcal{O}\left(\sigma^{2}+h^{2}\right)$,

where $\tilde{S}$ and $\tilde{S}_{h}$ shall denote the extension by zero. 
Proof. (1) Applying the Lemmata 3.7 and 3.39 as well as Theorem 3.2 immediately yields

$$
\begin{aligned}
\left\|S(\bar{q})-S_{h}\left(\bar{q}_{\sigma, h}\right)\right\|_{\mathrm{L}^{2}\left(\Omega_{0}\right)} & \leq\left\|S(\bar{q})-S\left(\bar{q}_{\sigma, h}\right)\right\|_{\mathrm{L}^{2}\left(\Omega_{0}\right)}+\left\|S\left(\bar{q}_{\sigma, h}\right)-S_{h}\left(\bar{q}_{\sigma, h}\right)\right\|_{\mathrm{L}^{2}\left(\Omega_{0}\right)} \\
& \leq c \cdot\left\|\bar{q}-\bar{q}_{\sigma, h}\right\|_{\mathrm{H}^{2}(I)}+c \cdot h^{2} \\
& =\mathcal{O}\left(\sigma^{2}+h^{2}\right) .
\end{aligned}
$$

(2) Again we split

$$
\begin{aligned}
& \left\|\tilde{S}(\bar{q}) \circ T_{\bar{q}}^{-1}-\tilde{S}_{h}\left(\bar{q}_{\sigma, h}\right) \circ T_{\bar{q}_{\sigma, h}}^{-1}\right\|_{\mathrm{L}^{2}(\hat{\Omega})} \leq\|\underbrace{\tilde{S}(\bar{q}) \circ T_{\bar{q}}^{-1}-\tilde{S}(\bar{q}) \circ T_{\bar{q}_{\sigma, h}}^{-1}}_{=e_{1}}\|_{\mathrm{L}^{2}(\hat{\Omega})} \\
& +\|\underbrace{S(\bar{q}) \circ T_{\bar{q}_{\sigma, h}}^{-1}-S\left(\bar{q}_{\sigma, h}\right) \circ T_{\bar{q}_{\sigma, h}}^{-1}}_{=e_{2}}\|_{\mathrm{L}^{2}\left(\Omega_{\bar{q}_{\sigma, h}}\right)} \\
& +\|\underbrace{S\left(\bar{q}_{\sigma, h}\right) \circ T_{\bar{q}_{\sigma, h}}^{-1}-S_{h}\left(\bar{q}_{\sigma, h}\right) \circ T_{\bar{q}_{\sigma, h}}^{-1}}_{=e_{3}}\|_{L^{2}\left(\Omega_{\bar{q}_{\sigma, h}}\right)} .
\end{aligned}
$$

and estimate each term separately. Because of the higher regularity of $\bar{q}$ (Thm. 3.16), Corollary 3.38 is also applicable to this continuous solution, hence for some $p>2$ it holds that $S(\bar{q}) \in \mathrm{W}^{2, p}\left(\Omega_{0}\right) \hookrightarrow \mathrm{C}^{1, \varepsilon}\left(\bar{\Omega}_{0}\right)$, i.e. $S(\bar{q})$ is Lipschitz continuous, and because of $S(\bar{q}) \in \mathrm{H}_{0}^{1}\left(\Omega_{0}\right)$ this is also true for $\tilde{S}(\bar{q})$. With Lemma 1.6 this now leads to

$$
\begin{aligned}
\left\|e_{1}\right\|_{\mathrm{L}^{2}(\hat{\Omega})}^{2} & \leq c \cdot\left\|e_{1}\right\|_{\mathrm{L}^{\infty}(\hat{\Omega})} \leq c \cdot\left\|T_{\bar{q}}^{-1}-T_{\bar{q}_{\sigma, h}}^{-1}\right\|_{\mathrm{L}^{\infty}(I)} \\
& \leq c \cdot\left\|\bar{q}-\bar{q}_{\sigma, h}\right\|_{\mathrm{H}^{2}(I)}=\mathcal{O}\left(\sigma^{2}+h^{2}\right) .
\end{aligned}
$$

For the second part we additionally use a transformation argument and get

$$
\begin{aligned}
\left\|e_{2}\right\|_{\mathrm{L}^{2}\left(\Omega_{\bar{q}_{\sigma}, h}\right)}^{2} & =\int_{\Omega_{\bar{q}_{\sigma, h}}}\left(S(\bar{q}) \circ T_{\bar{q}_{\sigma, h}}^{-1}-S\left(\bar{q}_{\sigma, h}\right) \circ T_{\bar{q}_{\sigma, h}}^{-1}\right)^{2} \mathrm{~d} x \\
& =\int_{\Omega_{0}}\left(S(\bar{q})-S\left(\bar{q}_{\sigma, h}\right)\right)^{2} \gamma_{\bar{q}_{\sigma, h}} \mathrm{~d} x \\
& \leq c \cdot\left\|S(\bar{q})-S\left(\bar{q}_{\sigma, h}\right)\right\|_{\mathrm{L}^{2}\left(\Omega_{0}\right)}^{2} \leq c \cdot\left\|\bar{q}-\bar{q}_{\sigma, h}\right\|_{\mathrm{H}^{2}(I)}^{2} \leq c \cdot\left(\sigma^{2}+h^{2}\right)^{2} .
\end{aligned}
$$

For the last part, $e_{3}$, we can proceed as in the previous step and also make use of Lemma 3.39.

\section{Numerical Results}

In this section the a priori error estimate from the previous section shall be verified by a numerical example. We therefore implemented the forms $a(\cdot)(\cdot, \cdot)$ and $l(\cdot)(\cdot)$ as well as the functional $J(\cdot, \cdot)$ in the optimization toolkit RoDoBo [2] and the finite element toolkit Gascoigne [1]. The derivatives of $j_{h}$ have been computed via a domain integral representation, and the optimal control problem (2.4) has been solved via a Newton method. For more details on Newton's method in the context of shape optimization we refer the reader to [21]. In [20] a general overview on numerical methods in shape optimization is given.

In the first example we chose

$$
\begin{aligned}
u_{d} & =\sin (\pi x) \cdot(1-y) \cdot(y+0.5 \sin (2 \pi x)), \\
f & =-\Delta u_{d}+u_{d},
\end{aligned}
$$

which means that the exact solution would have been $\bar{q}=-0.5 \sin (2 \pi x)$ in case of $\alpha=0$. 

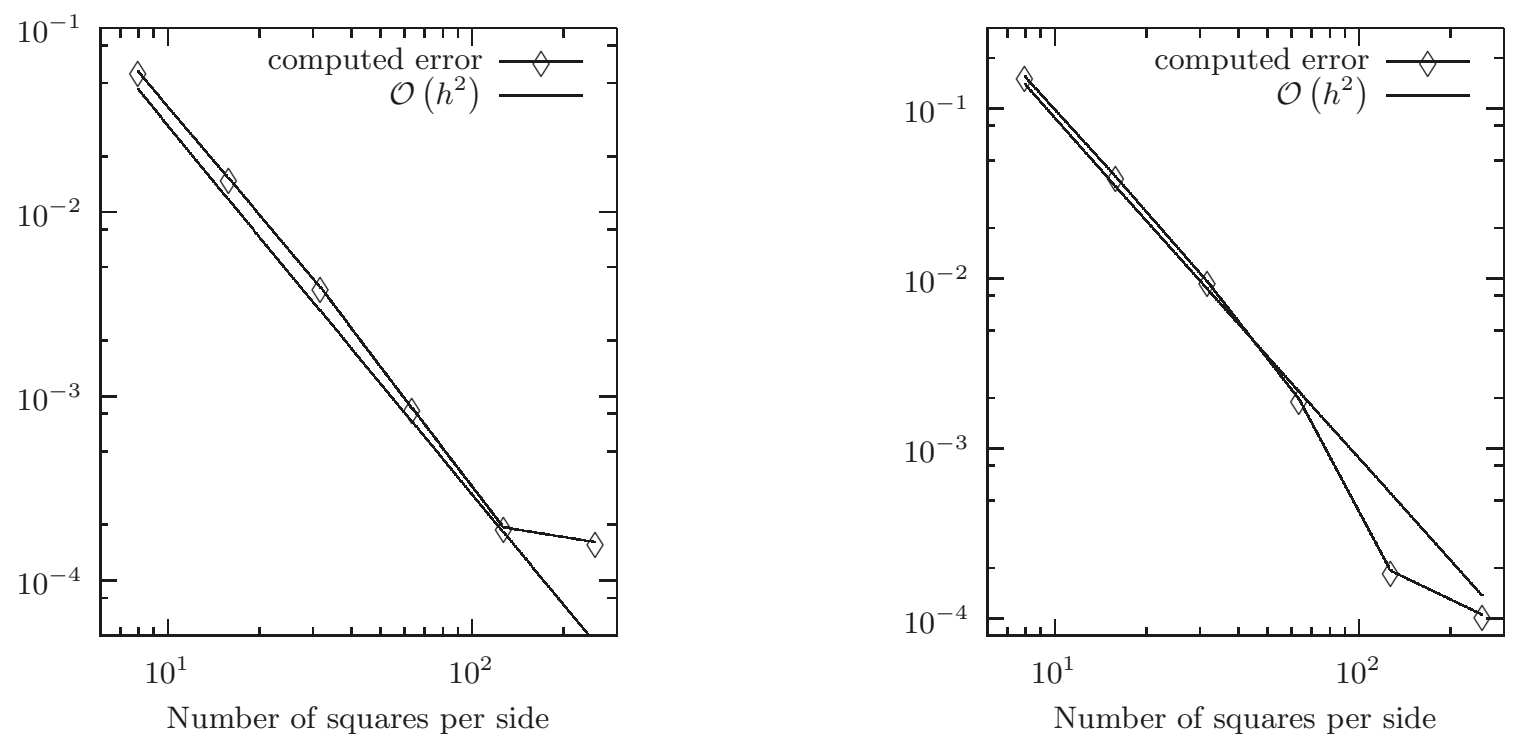

Figure 2. Discretization error $\left\|\bar{q}_{\sigma}-\bar{q}_{\sigma, h}\right\|_{\mathrm{H}^{2}(I)}$ for example (4.1) (left) and example (4.2) (right).
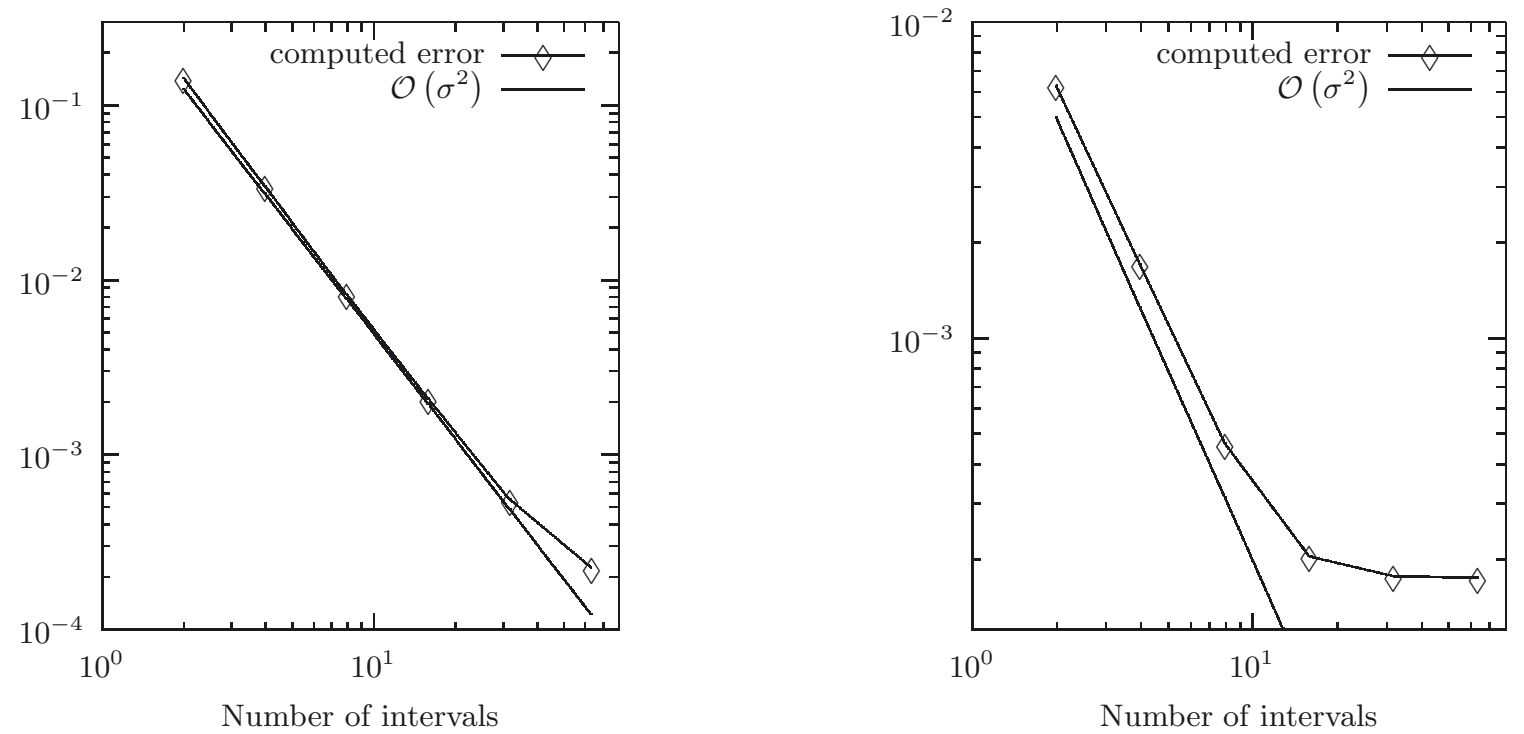

FiguRE 3. Discretization error $\left\|\bar{q}-\bar{q}_{\sigma}\right\|_{\mathrm{H}^{2}(I)}$ for example (4.1) (left) and example (4.2) (right).

In the second example we chose

$$
\begin{aligned}
u_{d} & =\sin (\pi x y) \cdot \sin \left(y^{2}\right), \\
f & =26 \exp \left(-14\left((x-0.8)^{2}+(y-1)^{2}\right)\right),
\end{aligned}
$$

with unknown exact solution. 
All the computations have been carried out with $\alpha=10^{-3}$. In order to illustrate the result of Theorem 3.2 numerically, we split the error: for the error in $h$ we computed, for a fixed $\sigma=60^{-1}$, a reference solution on a fine grid with $h=512^{-1}$. For the error in $\sigma$ we computed, for fixed $h=256^{-1}$, a reference solution with $\sigma=128^{-1}$. It can be seen that the error in $h$ as well as the error in $\sigma$ behave as predictet by Theorem 3.2. Due to the smoothing effect of the regularization term, $\|\bar{q}\|_{\mathrm{H}^{2}(I)}$ is very small in both examples. Hence, the optimal domains $\Omega_{\bar{q}}$ both look approximately like the unit square.

\section{REFERENCES}

[1] Gascoigne: The finite element toolkit. http://www.gascoigne.uni-hd.de/

[2] Rodobo: A ct+library for optimization with stationary and nonstationary pdes. http://rodobo.uni-hd.de/

[3] Y.A. Alkhutov and V.A. Kondrat' ev, Solvability of the Dirichlet problem for second-order elliptic equations in a convex domain. Differentsial' nye Uravneniya 28 (1992) 806-818, 917.

[4] A. Ambrosetti and G. Prodi, A Primer of Nonlinear Analysis, vol. 34, Cambridge Studies in Advanced Mathematics. Cambridge University Press, Cambridge (1993).

[5] D. Braess, Finite Elemente, Springer-Verlag (2007).

[6] E. Casas and F. Tröltzsch, Error estimates for the finite-element approximation of a semilinear elliptic control problem. Control Cybernet. 31 (2002) 695-712.

[7] E. Casas and F. Tröltzsch, A general theorem on error estimates with application to a quasilinear elliptic optimal control problem. Comput. Optim. Appl. 53 (2012) 173-206.

[8] D. Chenais and E. Zuazua, Controllability of an elliptic equation and its finite difference approximation by the shape of the domain. Numer. Math. 95 (2003) 63-99.

[9] D. Chenais and E. Zuazua, Finite-element approximation of 2D elliptic optimal design. J. Math. Pures Appl. 85 (2006) $225-249$.

[10] T. Dupont and R. Scott, Polynomial approximation of functions in Sobolev spaces. Math. Comput. 34 (1980) $441-463$.

[11] K. Eppler, H. Harbrecht, and R. Schneider, On convergence in elliptic shape optimization. SIAM J. Control Optim. 46 (2007) 61-83 (electronic).

[12] P. Grisvard, Elliptic problems in nonsmooth domains, vol. 24, Monographs and Studies in Mathematics, Pitman. Advanced Publishing Program, Boston, MA (1985).

[13] J. Haslinger and R.A.E. Mäkinen, Introduction to shape optimization. Theory, approximation, and computation, vol. 7, Advances in Design and Control, Society for Industrial and Applied Mathematics SIAM. Philadelphia, PA (2003).

[14] J. Haslinger and P. Neittaanmäki, Finite element approximation for optimal shape, material and topology design. John Wiley \& Sons Ltd., Chichester, 2nd edition (1996).

[15] K. Ito and K. Kunisch, Lagrange multiplier approach to variational problems and applications, vol. 15, Advances in Design and Control, Society for Industrial and Applied Mathematics. SIAM, Philadelphia, PA (2008).

[16] D.S. Jerison and C.E. Kenig, The Neumann problem on Lipschitz domains. Bull. Amer. Math. Soc. (N.S.) 4 (1981) $203-207$.

[17] D.S. Jerison and C.E. Kenig, The inhomogeneous Dirichlet problem in Lipschitz domains. J. Funct. Anal. 130 (1995) $161-219$.

[18] J. Kadlec, The regularity of the solution of the Poisson problem in a domain whose boundary is similar to that of a convex domain. Czechoslovak Math. J. 14 (1964) 386-393.

[19] K. Kunisch and G. Peichl, Numerical gradients for shape optimization based on embedding domain techniques. Comput. Optim. Appl. 18 (2001) 95-114.

[20] M. Laumen, A comparison of numerical methods for optimal shape design problems. Optim. Methods Softw. 10 (1999) $497-537$.

[21] M. Laumen, Newton's method for a class of optimal shape design problems. SIAM J. Optim. 10 (2000) 503-533 (electronic).

[22] J. Nečas, Sur la coercivité des formes sesquilinéaires, elliptiques. Rev. Roumaine Math. Pures Appl. 9 (1964) $47-69$.

[23] R. Rannacher and R. Scott, Some optimal error estimates for piecewise linear finite element approximations. Math. Comput. 38 (1982) 437-445.

[24] G. Savaré, Regularity results for elliptic equations in Lipschitz domains. J. Funct. Anal. 152 (1998) $176-201$.

[25] T. Slawig, Shape optimization for semi-linear elliptic equations based on an embedding domain method. Appl. Math. Optim. 49 (2004) 183-199.

[26] J. Sokołowski and J.-P. Zolésio, Introduction to shape optimization, Shape sensitivity analysis, vol. 16, Springer Series in Computational Mathematics. Springer-Verlag, Berlin (1992).

[27] F. Tröltzsch, Optimale Steuerung partieller Differentialgleichungen, Vieweg+Teubner (2009). 